\title{
Propagating vortices in ferrofluidic Couette flow under magnetic fields - Part I: Axial and symmetry breaking transversal orientated fields
}

\author{
Sebastian Altmeyer \\ Castelldefels School of Telecom and Aerospace Engineering, Universitat Politècnica de Catalunya, 08034 Barcelona, Spain
}

\section{A R T I C LE IN F O}

\section{Keywords}

Taylor-Couette flow

Ferrofluid

Axial and transverse magnetic fields

Symmetry breaking

Propagating vortices

\begin{abstract}
A B S T R A C T
Numerical investigations of propagating vortex states (pVs) for ferrofluidic Couette flow with small aspect ratio and fixed non-rotating end-walls are presented. We study structural modifications and changes in spatial and temporal behavior for $\mathrm{pV}$ solutions. The system is subjected to either pure axial or pure transverse magnetic field, with the latter already breaking the basic system symmetries. While under axial magnetic field pVs remain basically the same, they are found to appear in various configurations with different symmetry variation in flow structure and flow dynamics under symmetry breaking transversal magnetic field. pVs appearing in a pitchfork bifurcation are found either symmetric or asymmetric/alternating, regarding the full cycle of vortex generation, propagation and annihilation, in upper and lower system half.
\end{abstract}

\section{Introduction}

The flow confined between two concentric cylinders rotating with different velocity, Taylor-Couette flow, has been for more than a century the focus of scientific interest to study fundamental fluid dynamics, non-linear dynamics, self-organization, various hydrodynamic stabilities and pattern formation etc., both numerically and experimentally $[1,2]$. Although, classical fluids in this system setup (Taylor-Couette system, TCS) [3-5] have been studied for several decades the dynamics of complex fluids, e.g., ferrofluids [6,7] (manufactured fluids consisting of dispersion of magnetized nanoparticles in a liquid carrier), have attracted attention mainly in recent years/modern era [8-20].

In experimental realizations of Couette-flow, the viscous fluid is often enclosed by non-rotating axial end walls (e.g. non-rotating lids) at top and bottom, and therefore the axial translation invariance of the idealized infinite (periodic system) is broken [21]. At any driving rate, these end walls generate disturbances [22-25], resulting in an axisymmetric, secondary circulation, so-called Ekman vortices which overlay and deform idealized/theoretical Circular-Couette flow (CCF) and create a new stationary, rotationally symmetric basic flow [25].

Consider a ferrofluid, further parameters, such as the orientation of an applied magnetic field with respect to the fluid flow become important as this has crucial influence to the magnetoviscous effect in ferrofluids $[26,6]$. In general, any external applied magnetic field, independent of it's orientation, results in a stabilization of the basic state as well as shifting bifurcation thresholds for any flow structure [8,13-15]. For axial, radial or azimuthal orientated magnetic fields, the resulting effects are only quantitative and appear as differences/changes in the distance of the up-shift of primary bifurcation thresholds
$[8,13,15,26-28]$. However, the scenario is more complicate if a transversal field component is present. Aside the shift of bifurcation thresholds, such a field results in significant qualitative differences, also breaking the classical system symmetries for TCS $[13,16,17]$, rendering all flows to be three-dimensional and therefore increasing the already huge number of flow states known to exist in the system [1-5].

Although propagating vortex pattern are nothing uncommon and in fact appear in huge variety in TCS, the here studied propagating vortices (pVs) are special/different under certain aspects. Usually propagating structures in TCS include an azimuthal rotation, e.g. spiral vortices [21,4], wavy Taylor vortices [31-33], etc. By contrast here investigated pVs don't include any azimuthal rotation, only axial motion is involved. For classical TCS and pure axial field these are "M $=0$ "-mode $[29,30]$ solutions.

Among others, the present study has been motivated by the recent work of Ilzig et al. [30] who were the first to study pVs in a ferrofluid Couette flow. They investigated pVs in a ferrofluid with asymmetric axial boundary condition (one side open) exposed to external homogeneous axial magnetic fields. They detected an increasing orbital frequency (i.e. decreasing period time) with increasing field strengths as well as appearance of disturbed pVs due to temporarily superimposed spiral structures. However they could not provide deeper inside of the underlying topology of $\mathrm{pV}$ states.

The overall goal of this study (first and second part) is to expand the parameter range in which the $\mathrm{pV}$ states are existing, stable and unstable, and further to investigate the underlying topology of these flow states in ferrofluids in the presence of different magnetic fields. The current paper as the first part is focussed on either pure axial or pure transversal magnetic fields, with the latter already breaking the basic system symmetries. The second part [34] focus on oblique magnetic 
fields as superposition of axial and transversal magnetic fields, which results in complexer non-linear mode interaction and further modification of system symmetries, dynamics and topology.

\section{Methods}

\subsection{System setting and the Navier-Stokes equation}

We consider a standard Taylor-Couette system (TCS) (Fig. 1) consisting of two concentric, independently rotating cylinders. Within the gap between the two cylinders there is an incompressible, isothermal, homogeneous, mono-dispersed ferrofluid of kinematic viscosity $v$ and density $\rho$. The inner and outer cylinders have radius $R_{i}$ and $R_{o}$, and they rotate with the angular velocity $\omega_{i}$ and $\omega_{o}$, respectively. Here, we consider rigid boundary conditions in the axial direction with stationary non-rotating lids and no-slip boundary conditions on the cylinders. The height-to-gap aspect ratio is fixed to $\Gamma=L / d=4$. The system can be characterized in the cylindrical coordinate system $(r, \theta, z)$ by the velocity field $\mathbf{u}=(u, v, w)$ and the corresponding vorticity field $\nabla \times \mathbf{u}=(\xi, \eta, \zeta)$. The radius ratio of the cylinders, $R_{i} / R_{o}$ is kept fixed at 0.5 . A homogeneous magnetic field $\mathbf{H}=H_{x} \mathbf{e}_{x}\left[H_{z} \mathbf{e}_{z}\right]$ with a transversal component $H_{x}$ or an axial component $H_{z}$ is considered. [ $H_{z}$ and $H_{x}$ being the field strengths.] Length and time scales of the system are set by the gap width $d=R_{o}-R_{i}$ and the diffusion time $d^{2} / v$, respectively. The pressure in the fluid is normalized by $\rho v^{2} / d^{2}$, and the magnetic field $\mathbf{H}$ and the magnetization $\mathbf{M}$ can be conveniently normalized by the quantity $\sqrt{\rho / \mu_{0}} v / d$, with free space permeability $\mu_{0}$. These considerations lead to the following set of non-dimensionalized hydro-dynamical equations $[17,35]$ :

$$
\begin{aligned}
\left(\partial_{t}+\mathbf{u} \cdot \nabla\right) \mathbf{u}-\nabla^{2} \mathbf{u}+\nabla p & =(\mathbf{M} \cdot \nabla) \mathbf{H}+\frac{1}{2} \nabla \times(\mathbf{M} \times \mathbf{H}), \\
\nabla \cdot \mathbf{u} & =0 .
\end{aligned}
$$

On the cylindrical surfaces, the velocity fields are given by $\mathbf{u}\left(r_{i}, \theta, z\right)=\left(0, R e_{i}, 0\right)$ and $\mathbf{u}\left(r_{o}, \theta, z\right)=\left(0, R e_{o}, 0\right)$, where the inner and outer Reynolds numbers are $R e_{i}=\omega_{i} r_{i} d / v$ and $R e_{o}=\omega_{o} r_{o} d / v$, respectively, where $r_{i}=R_{i} /\left(R_{o}-R_{i}\right)$ and $r_{o}=R_{o} /\left(R_{o}-R_{i}\right)$ are the non-dimensionalized inner and outer cylinder radii, respectively. In the pre-

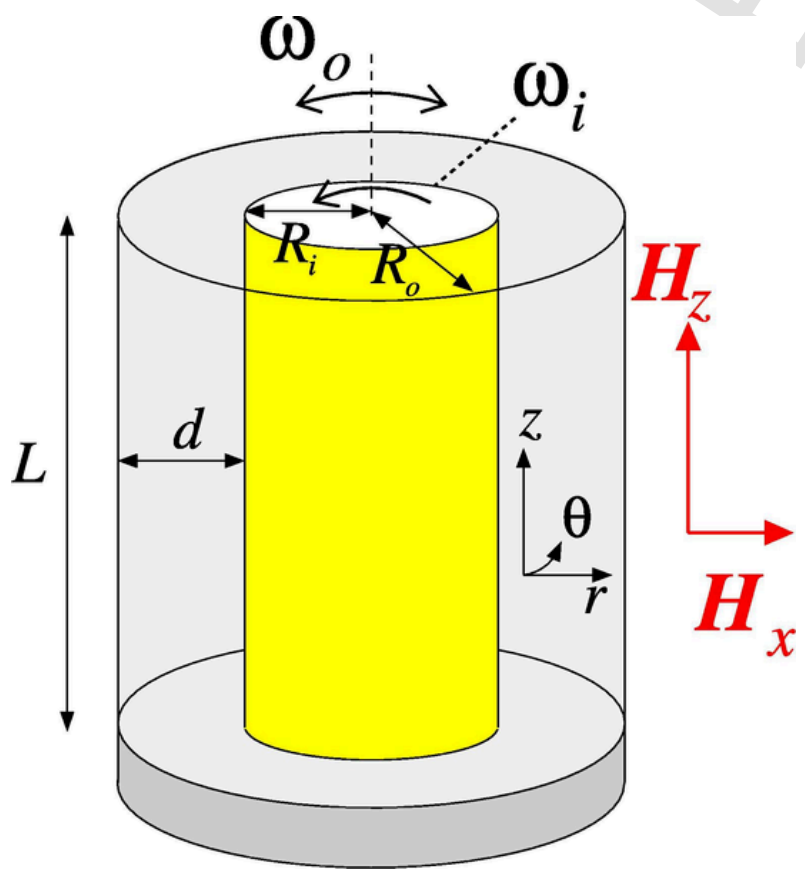

Fig. 1. Schematic of the Taylor-Couette system (TCS) with an external applied homogeneous transversal [axial] magnetic field $H_{e x t}=H_{x} e_{x}\left[H_{z} e_{z}\right]$. sent work we consider counter-rotating cylinders and therefore keep them fixed to $R e_{i}=195$ and $R e_{o}=-300$, respectively, meaning a rotation ratio $R e_{o} / R e_{i} \approx-1.54$. Thereby the chosen fixed values of $R e_{i}$ and $R e_{o}$ guarantee the flow to remain supercritical for sufficient parameter range $s_{x}, s_{z}$, as it is a matter of fact that any magnetic field (independent it's orientation) stabilizes the basic state, i.e. shifting the bifurcation thresholds to larger control parameters.

Eq. (1) is to be solved together with an equation that describes the magnetization of the ferrofluid. Using the approach of Niklas [8], derived from the theory by Shliomis [6] under the assumption of a stationary magnetization, and some further simplifications (see Appendix for details) leads to the following ferrohydrodynamical equations.

$$
\begin{aligned}
\left(\partial_{t}+\mathbf{u} \cdot \nabla\right) \mathbf{u}-\nabla^{2} \mathbf{u}+\nabla p_{M} & \\
= & s_{N}^{2}\left\{\nabla^{2} \mathbf{u}-\frac{4}{5}[\nabla \cdot(\mathbb{S H})]-\mathbf{H}\right. \\
& \times\left[\frac{1}{2} \nabla \times(\nabla \times \mathbf{u} \times \mathbf{H})-\mathbf{H} \times\left(\nabla^{2} \mathbf{u}\right)+\frac{4}{5} \nabla\right. \\
& \times(\mathbb{S H})]\},
\end{aligned}
$$

$\mathbb{S}$ is the symmetric component of the velocity gradient tensor $[17,35]$. Thus the effect of the magnetic field and the magnetic properties of the ferrofluid on the velocity field can be characterized by a single parameter, the magnetic field or the Niklas parameter [8]:

$s_{N}^{2}=s_{x}^{2}+s_{z}^{2}$,

with

$s_{x}^{2}=\frac{2(2+\chi) H_{x} c_{N}}{(2+\chi)^{2}-\chi^{2} \eta^{2}}, s_{z}^{2}=H_{z} c_{N}$.

Here, $\chi$ is the magnetic susceptibility of the ferrofluid, which can be approximated by Langevin's formula [36], and the Niklas coefficient $c_{N}$ depends on the properties of the ferrofluid and magnetic field $[8,13,14]$ as described in the Appendix. The ferrohydrodynamic system Eq. (2) is solved numerically with the code G1D3 [6]. G1D3 combines a finite difference method of second order in $(r, z)$ and time (explicit) with spectral decomposition in $\theta$. The numerical approach to solving the equations is outlined in more detail the Appendix.

In this paper we present results in absence (none) of any applied magnetic field, $\left(s_{x} ; s_{z}\right)=(0.0 ; 0.0)$, pure transverse magnetic field, $\left.\left.\left(s_{x} \in\right] 0,1\right] ; 0.0\right)$, and pure axial magnetic field, $\left.\left.\left(0.0 ; s_{z} \in\right] 0,1\right]\right)$. These values/parameters correspond to moderate magnetic fields used in several experiments $[9,15,16]$.

\subsection{Symmetries}

In a classical TCS or a ferrofluidic TCS without any external magnetic field where the fluid is confined by end walls, the system is invariant with respect to arbitrary rotations about the axis and the reflections about axial mid-height. For a ferrofluid under a transverse magnetic field, these symmetries are broken and the flow is inherently three-dimensional for any non-zero values of the parameters $R e_{i}, R e_{o}$ and $s_{x}$ , due to the rotation of the cylinders $[13,14,16,17,37,28]$. With at least one cylinder rotating, the inclusion of the magnetic terms in the ferro-hydrodynamic equation results in a downward directed force on the side where the field enters the system $(\theta=0)$, and an upward directed force on the opposite side $(\theta=\pi)$ where the field exits the annulus. The resulting flow states can possess more complicated symmetries, such as the reflection $K_{z}^{H_{x}}$ about the annulus mid-height plane along with an inversion of the magnetic field direction. There can also be a rotational invariance $R_{\alpha}^{H_{x}}$ for discrete angle $\alpha=\pi$ in combination with the reversal of the magnetic field, where the angle $\pi$ specifies the direction of the magnetic field when entering the annulus [17]. Thus the 
symmetries associated with the velocity field are

$R_{\pi}^{H_{x}}\left(u, v, w, H_{x}\right)(r, \theta, z, t)=\left(u, v, w,-H_{x}\right)(r, \theta+\pi, z, t)$,

$K_{z}^{H_{x}}\left(u, v, w, H_{x}\right)(r, \theta, z, t)=\left(u, v,-w,-H_{x}\right)(r, \theta,-z, t)$.

Given a periodic solution (with period $\tau$ ), the flow field is also invariant under the discrete time translation

$\Phi_{\tau}\left(u, v, w, H_{x}\right)(r, \theta, z, t)=\left(u, v, w, H_{x}\right)(r, \theta, z, t+\tau)$.

Thus the symmetry group of classical TCS with stationary lids, $S O(2) \times Z_{2} \times \mathbb{R}$ is replaced by complexer discrete spacetime symmetries. The full symmetry group of the problem under transverse magnetic field reads as $S O(2)^{H_{x}} \times Z_{2}^{H_{x}} \times \mathbb{R}$. Further details of the magnetic field induced twofold symmetry can be found in Ref. [17].

\subsection{Nomenclature/Notation \& parameter space}

The present study focus on flow dynamics in TCS with relative small aspect-ratio $\Gamma=4.0$ and counter-rotating cylinders for fixed outer Reynolds number $R e_{o}=-300$ and fixed inner Reynolds number $R e_{i}=195$ (i.e. rotation ratio equal $R e_{o} / R e_{i} \approx-1.54$ ), respectively. As a result common appearing structures in absence of any magnetic field are (pure $m=0$ mode) flow states, either stationary solution $6 \mathrm{~V}$, (i.e. 6-cell flow $[29,38,39])$ as well as periodic, axial propagating solutions, so called $\mathrm{pV}$ states $[29,30]$. These $\mathrm{pV}$ states illustrate a spatio-temporal dynamics with periodic vortex generation and annihilation. Worth to mention that the here studied parameter regime is well below the marginal (linear) stability thresholds of corresponding modes. Table 1 provides an overview of all different flow states discussed in this work. Acronyms, flow states, including main characteristics, dominant modes, numbers (\#) of present vortex cells, flow dynamics, magnetic field correlation and classification are indicated. Worth to mention, that former reported propagating vortex states $[29,30]$ were just denoted as $\mathrm{pV}$ states. However, based on further characteristics we will basically distinguish between $\mathrm{pV}^{s}$ and $\mathrm{pV}^{a}$, based on the symmetry of the vortex propagating structures with respect to system mid-hight. See below for further details.

Either for pure axial and transversal magnetic field all time dependent solutions of propagating flow states (pV) appear as limit cycle solution. Worth to mention that for oblique fields (combination of both) the scenario becomes more difficult and pVs exist with higher complexity with respect to the underlying manifold. This is focus of the second part of this study [34].

\section{Results}

Before we start the discussion of effects on $\mathrm{pV}$ states with variation any magnetic field strength, we shortly describe the $\mathrm{pV}$ state that appears at given parameters in absence of any field. For further details we refer to [29] in which pVs in classical TCS have been studied numerically and experimentally in detail for various aspect ratios.

\subsection{Propagating Vortex flow in absence of any magnetic field}

Fig. 2 shows snapshots over one period $\tau \approx 0.173$ of $\mathrm{pV}^{\mathrm{s}}$ in absence of any applied magnetic field. At (a) $t=0$ the $\mathrm{pV}^{\mathrm{s}}$ state exhibits six vor-

Table 1

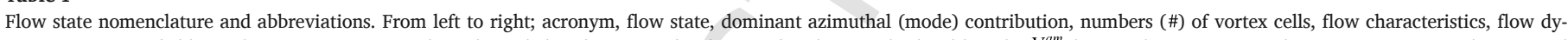

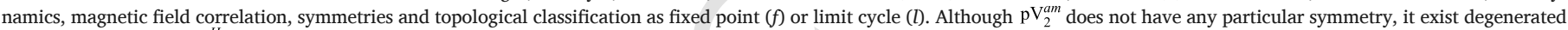
together with $\mathrm{pV}_{2}^{a m *}=K_{z}^{H_{X}} p V_{2}^{a m}$.

\begin{tabular}{|c|c|c|c|c|c|c|c|c|}
\hline Acronym & Flow state & Modes $m$ & \# vortex cells & Characteristics & Dynamics & $H$ field & Symmetries & Solution \\
\hline $6 \mathrm{~V}$ & Normal 6-cell & 0 & 6 & stationary & - & $s_{x}=0$ & $K_{z}$ & $f$ \\
\hline $6 \mathrm{~V}_{2}$ & Normal 6-cell & 0,2 & 6 & stationary & - & $s_{x} \neq 0$ & $K_{z}^{H_{x}}$ & $f$ \\
\hline $\mathrm{pV}^{\mathrm{s}}$ & Propagating Vortex & 0 & $6+n, n \in\{2 \mathbb{N}\}$ & periodic & symmetric prop. & $s_{x}=0$ & $K_{z}$ & $l$ \\
\hline $\mathrm{pV}_{2}^{s}$ & Propagating Vortex & 0,2 & $6+n, n \in\{2 \mathbb{N}\}$ & periodic & symmetric prop. & $s_{x} \neq 0$ & $K_{z}^{H_{x}}$ & $l$ \\
\hline $\mathrm{pV}_{2}^{a}$ & Propagating Vortex & 0,2 & $6+n, n \in\{2 \mathbb{N}\}$ & periodic & alternate prop. & $s_{x} \neq 0$ & $S^{H_{x}}$ & $l$ \\
\hline $\mathrm{pV}_{2}^{a m}$ & Propagating Vortex & 0,2 & $6+n, n \in\{2 \mathbb{N}\}$ & periodic & alternate prop. & $s_{x} \neq 0$ & - & $l$ \\
\hline
\end{tabular}
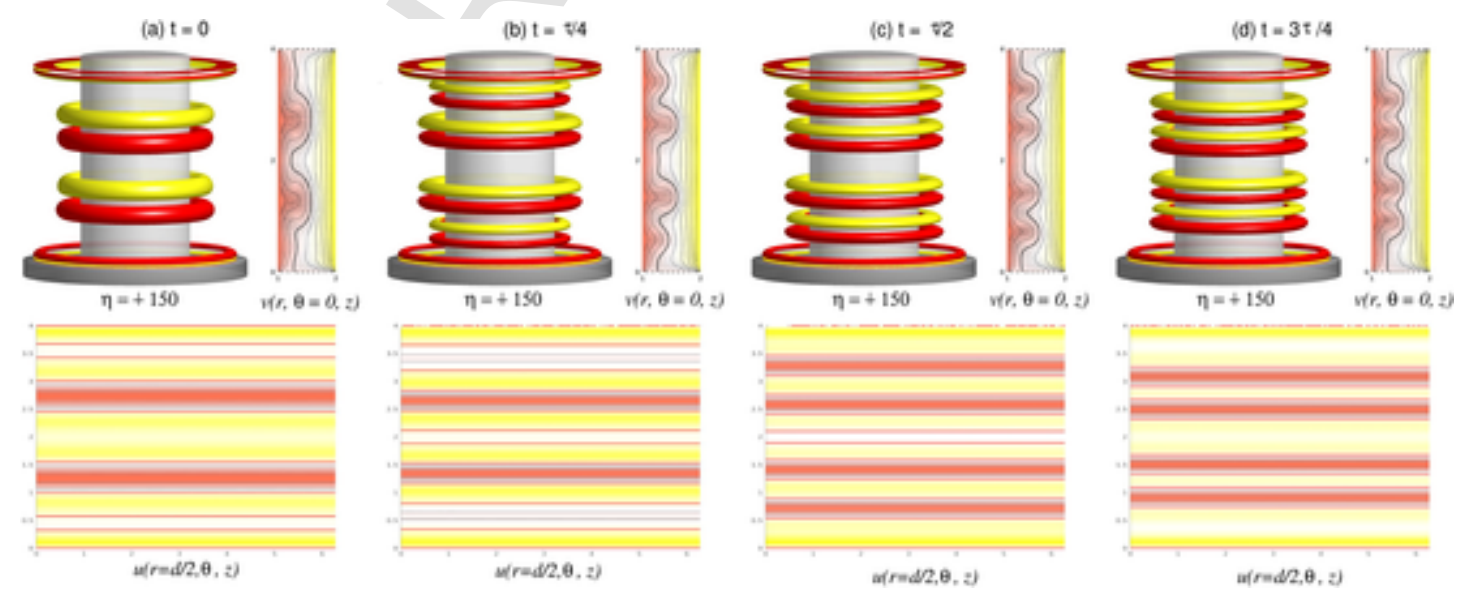

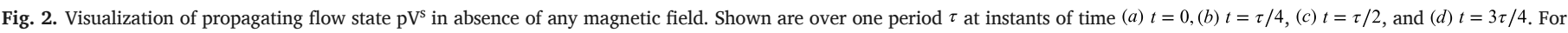

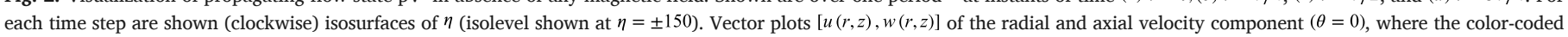

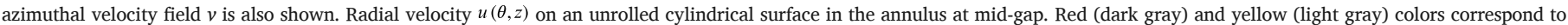

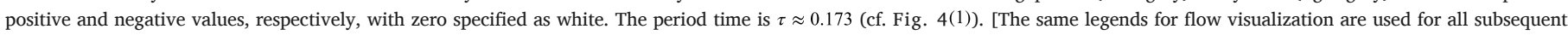

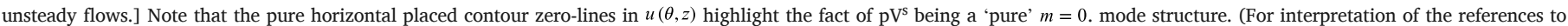
colour in this figure legend, the reader is referred to the web version of this article.) 


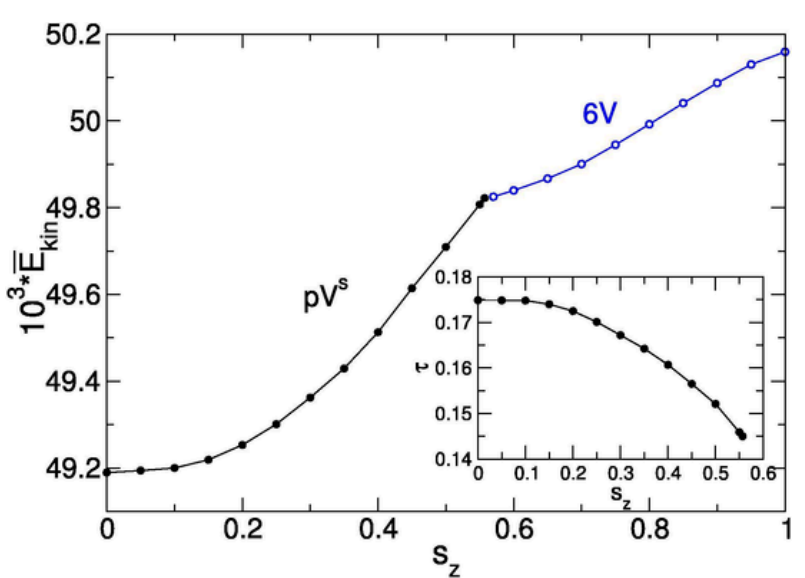

Fig. 3. Bifurcation diagram and period time evolution with $s_{z}$. Shown is the total (time-averaged for time-dependent flow solutions) modal kinetic energy $\bar{E}_{k i n}$. Solid [open] symbols indicate time dependent [stationary] solutions. The inset illustrates the variation of period time $\tau$ with $s_{z}$ for $\mathrm{pV}^{\mathrm{s}}$ states.

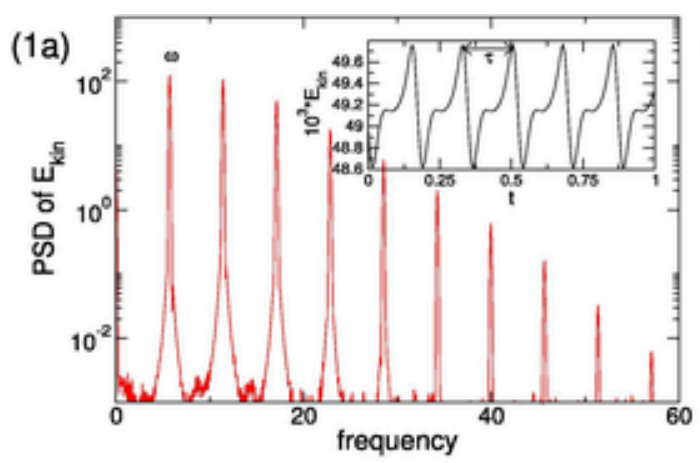

(2a)
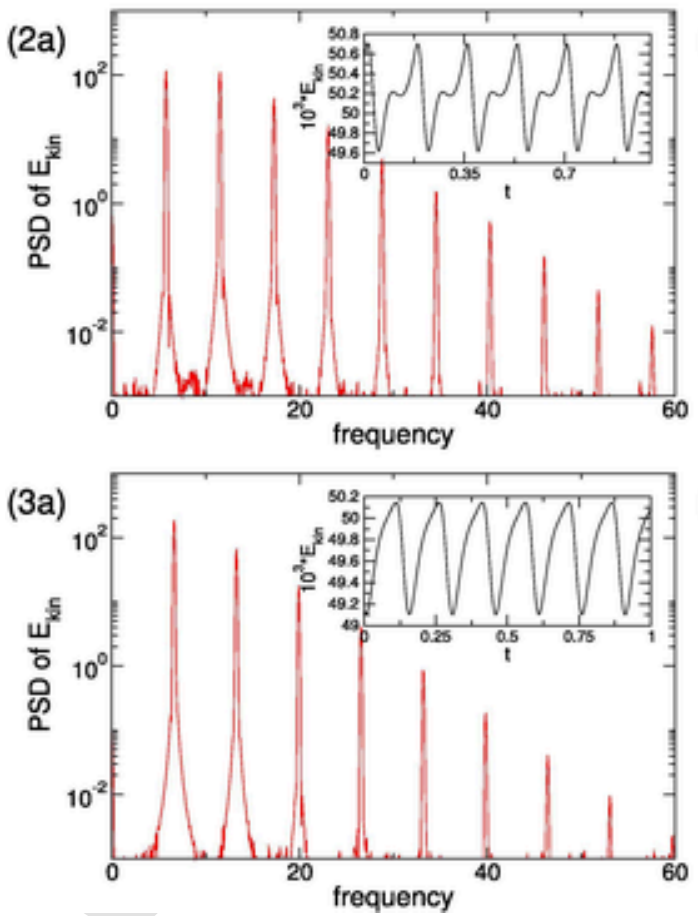

tices very similar to the stationary $6 \mathrm{~V}$ state (i.e. two pairs of vortices in the bulk plus two Ekman vortices near the lids). Moving forward in time $(b)$ two new vortex pairs are generated simultaneously and symmetrically near both, the upper and lower Ekman vortices creating a temporarily state with higher number of vortices (here 10). These new vortex pairs grow and propagate towards the mid-height of the system where they finally become annihilated and the flow returns to the initial $\mathrm{pV}^{\mathrm{s}}$ state exhibiting six vortices. Another perspective of this periodic motion of vortices towards the center is given by the spacetime plots of the radial velocity in Fig. 5(a). The black zero-contour lines clearly identify the dynamics as well as the $K_{z}$ symmetry of $\mathrm{pV}^{\mathrm{s}}$. For more details, see also Fig. 2 and corresponding description in [29]. Worth to emphasis that for $\mathrm{pV}^{\mathrm{s}}$ the process of generation and annihilation appears simultaneously in either upper and lower half of the system. The fact $\mathrm{pV}^{\mathrm{s}}$ being pure a 'pure' $m=0$ mode structure $[13,15,29,30]$ is clearly visible in the pure horizontal placed contour zero-lines in $u(\theta, z)$ (Fig. 2).

Corresponding power spectral densities (PSDs) and time series of $E_{k i n}$ and $\eta_{ \pm}$for $\mathrm{pV}^{\mathrm{s}}$ are shown in Fig. 4(1). Note that here $\tau$ is the same period of the time series of $E_{k i n}$ and $\eta_{ \pm}$as the vortices are simultaneously and symmetrically propagating from the lids towards mid-height.
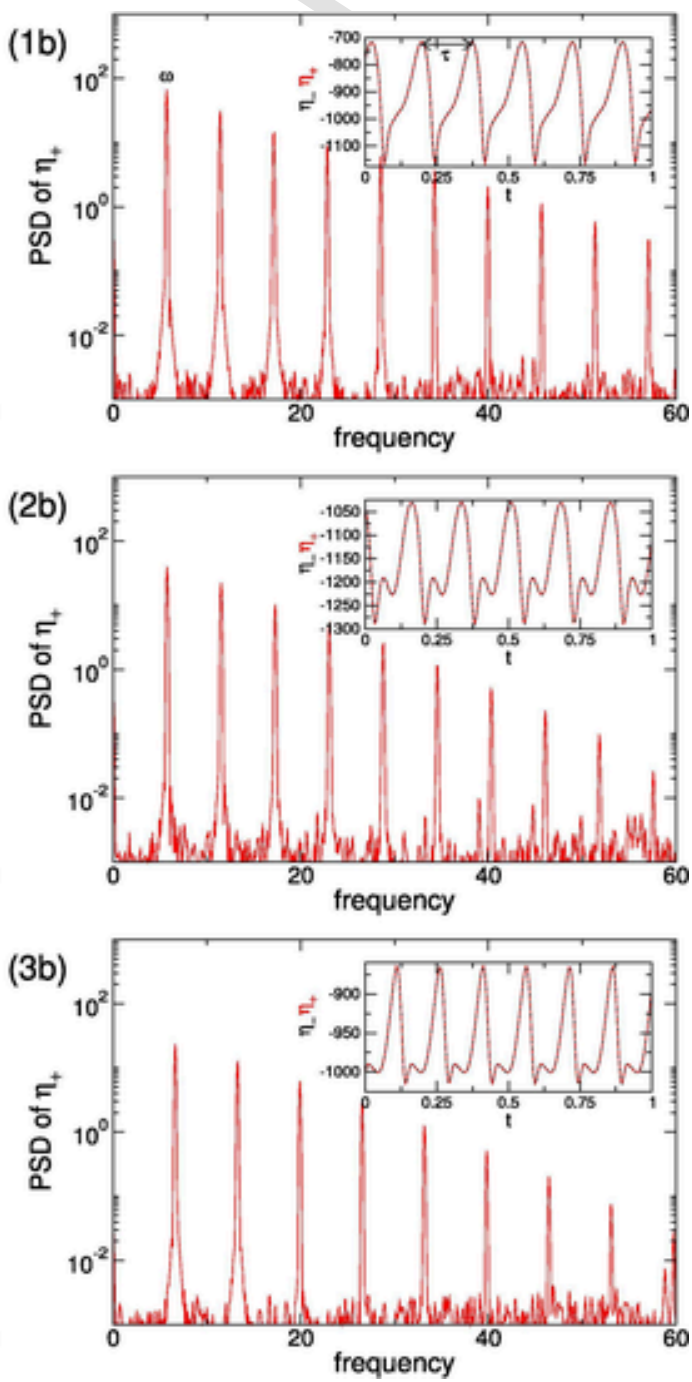

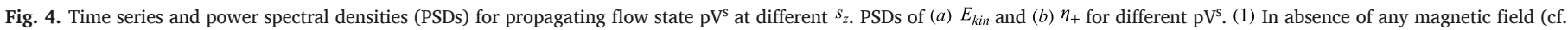

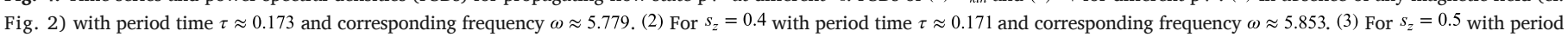

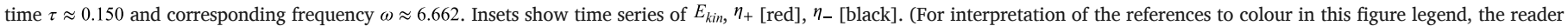
is referred to the web version of this article.) 

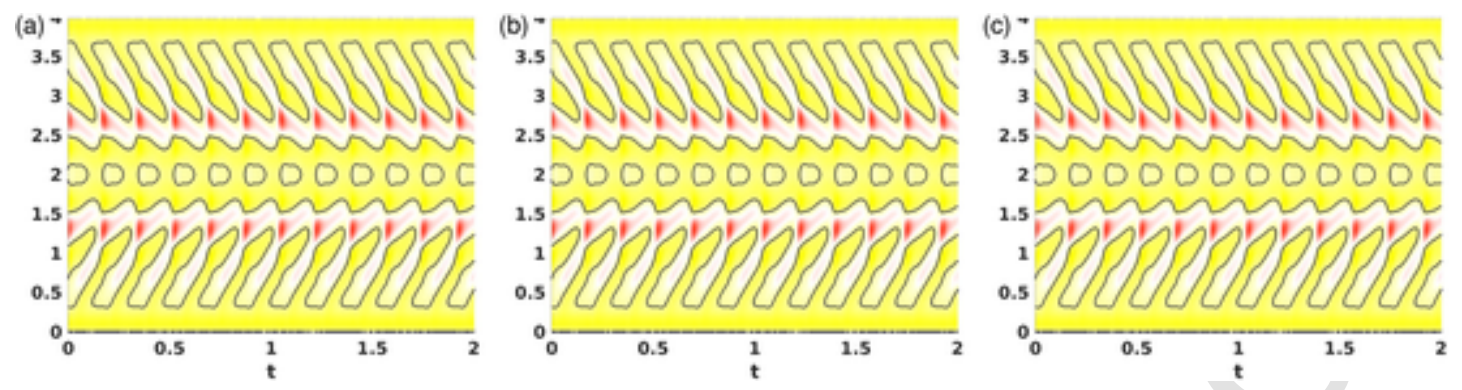

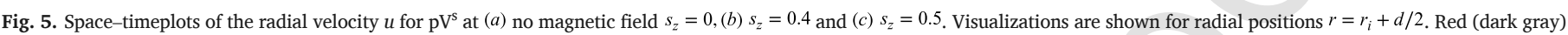

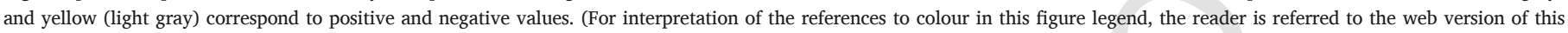
article.)

\section{Propagating Vortex flow in axial magnetic field}

As mentioned before, this work, among others was motivated by the recent experimental work by Ilzig et al. [30]. They studied pVs under axial magnetic field for aspect ratios $\Gamma=5$ (odd) and $\Gamma=6$ (even). However, their work dealt with different boundary conditions, as they used a one side open system and therefore the basic system symmetries are intrinsically broken. In addition the present study tends more to focus on quantitative analysis, bifurcation scenario and underlying topology. Following we start considering a pure axial magnetic field and variation its field strength $s_{z}$.

\subsection{Bifurcation scenario and period time with $s_{z}$}

The bifurcation diagram with $s_{z}$ is shown in Fig. 3, including an inset illustrating corresponding evolution in period time $\tau$ for $\mathrm{pV}^{\mathrm{s}}$. With increasing the field strength, $s_{z}$, the modal kinetic (time-averaged) energy $\bar{E}_{k i n}$ for $\mathrm{pV}^{\mathrm{s}}$ grows monotonously, first moderate (until $s_{z} \approx 0.2$ ) and with larger field strength faster before $\mathrm{pV}^{s}$ eventually disappear at $s_{z} \approx 0.565$ leaving a stationary $6 \mathrm{~V}$ state behind. Although a change in slope is clearly visible after reaching the stationary $6 \mathrm{~V}$ state, the general monotonously increasing trend in $\bar{E}_{k i n}$, with increasing $s_{z}$, remains unchanged. In parallel, the corresponding period time $\tau$ behaves just opposite and decreases monotonously with $s_{z}$ before $\mathrm{pV}^{\mathrm{s}}$ vanishes (inset in Fig. 3). This behavior is congruent with the well known stabilization effect of magnetic fields $[8,13,15]$ which means an effective shift of the marginal stabilization threshold to larger control parameters. Ilzig et al. [30] detected the same characteristics in their experimental study, which they described as change in orbital frequency of pVs. Important to mention that over the whole here investigated parameter space $s_{z} \in[0 ; 1]$ the symmetry of all flow structures, either time-dependent $\mathrm{pV}^{\mathrm{S}}$ and stationary $6 \mathrm{~V}$ remain the same, all fulfill $K_{z}$ symmetry.

More general speaking, due to the stabilization effect, an increase in the magnetic field strength (independent of the field direction) has qualitative a similar effect, e.g. decreasing $R e_{i}$, as another control parameter $[13,15]$.

For increasing $s_{z}$, the time series of $E_{k i n}$ as global measure simplify, while the corresponding ones of $\eta_{ \pm}$become more pronounced (insets in Fig. 4). Meanwhile the general dynamics for $\mathrm{pV}^{\mathrm{s}}$ remain unchanged by

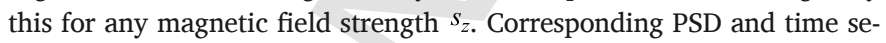
ries for both, global measure $E_{k i n}$ and local measures $\eta_{ \pm}$illustrate the dynamics to be associated with one single frequency $\omega$ (or corresponding period time $\tau$, cf. inset in Fig. 4(1a)) rendering the flow to be a limit cycle solution. This, together with the decreasing time period $\tau$ can be also spotted in the spacetime plots in Fig. 5, which provide another perspective of the global flow dynamics. The zero-contour level is in black and indicates how the new generated vortex pairs travel (from the appearance near both lids) towards the system mid-height where they eventually become annihilated. The flow dynamics becomes less intense (decreasing amplitudes) with the propagation region shrinking in size (z-direction) towards the onset of $\mathrm{pV}^{\mathrm{s}}$ at $s_{z} \approx 0.565$. In general, the global flow dynamics becomes smoother with increasing moving closer to the onset as indicated in simpler time series of $E_{k i n}$ (Fig. 4(3a)) and less sharp separation between the zero-contour lines identifying consecutive periods (Fig. $5(b, c)$ ). Aside the $K_{z}$ symmetry is obvious in the spacetime plots.

With increasing $s_{z}$ the basic flow dynamics for $\mathrm{pV}^{s}$ remain unchanged, with only qualitative measure in slightly shorter period time. In particular the flow remains $2 \mathrm{D}$ being a 'pure' $m=0$ mode structure $[13,15,30]$. An analog flow visualizations to Fig. 2, but for $\mathrm{pV}^{\mathrm{s}}$ at $s_{z}=0.5$ is provided in Fig. 1 in Supplementary Materials (SM) and highlights this characteristic with clear visible horizontal contour zero-lines in $u(\theta, z)$.

All our finding for pure axial magnetic fields are in qualitative good agreement with the experimental findings by Ilzig et al. [30] despite their study of a one side open system (with different boundary conditions). A qualitative comparison to their experimental results is provided in SM.

\subsection{Space time evolution and phase Space with $s_{z}$}

In order to visualize the change in flow dynamics with variation in $s_{z}$ , Fig. 6 illustrate the phase portraits of $\mathrm{pV}^{\mathrm{s}}$ solutions for different $s_{z}$ over both, $(a)\left(\eta_{+}, \eta_{-}\right)$and $(b)\left(\eta_{ \pm}, E_{k i n}\right)$ planes. In the $\left(\eta_{+}, \eta_{-}\right)$plane all $\mathrm{pV}^{\mathrm{s}}$ states come to lie on the diagonal $\eta_{+}=\eta_{-}$line, i.e. topological speaking a degenerated limit cycle. The distance from the phase portraits to the diagonal line $\eta_{-}=\eta_{+}$is a measure of the degree to which $Z_{2}$ symmetry is broken However, the $\left(\eta_{ \pm}, E_{k i n}\right)$ plane clearly illustrates the limit cycle characteristic of $\mathrm{pV}^{\mathrm{s}}$ together with the coincide shrinking of the cycles with increasing $s_{z}$. Moving towards the onset the region explored by corresponding trajectories shrink until it eventually collapsing into a single fixed point, the stationary solution $6 \mathrm{~V}$ at $s_{z} \approx 0.58$.

Finally Fig. 7 illustrates this stationary solution $6 \mathrm{~V}$ at $s_{z}=0.6$, close to the onset of $\mathrm{pV}^{\mathrm{s}}$. Either flow visualizations and mode amplitudes $\left|u_{m, n}\right|$ (Fig. $7(e)$ ) highlight the 2D character of the flow state ( $m=0$ flow [13,30], all larger modes $m \geqslant 1$ are identical zero).

\section{Propagating Vortex flow in transverse magnetic field}

As discussed in Section 2.2 any transverse field component destroys the basic system symmetries (invariant to arbitrary time translation, rotations about the axis, reflections about axial mid-height [40]), and replace them by complexer symmetries (see also Eq. (5)) [17]. As a result also the propagating vortices lose their $2 \mathrm{D}$ characteristics and become inherentially 3D for $s_{x} \neq 0$, which we indicate as $\mathrm{pV}_{2}$. The index 2 is used to indicate the stimulation of $m=2$ modes due to a symmetry breaking transversal magnetic field $[13,16,17,28]$. 

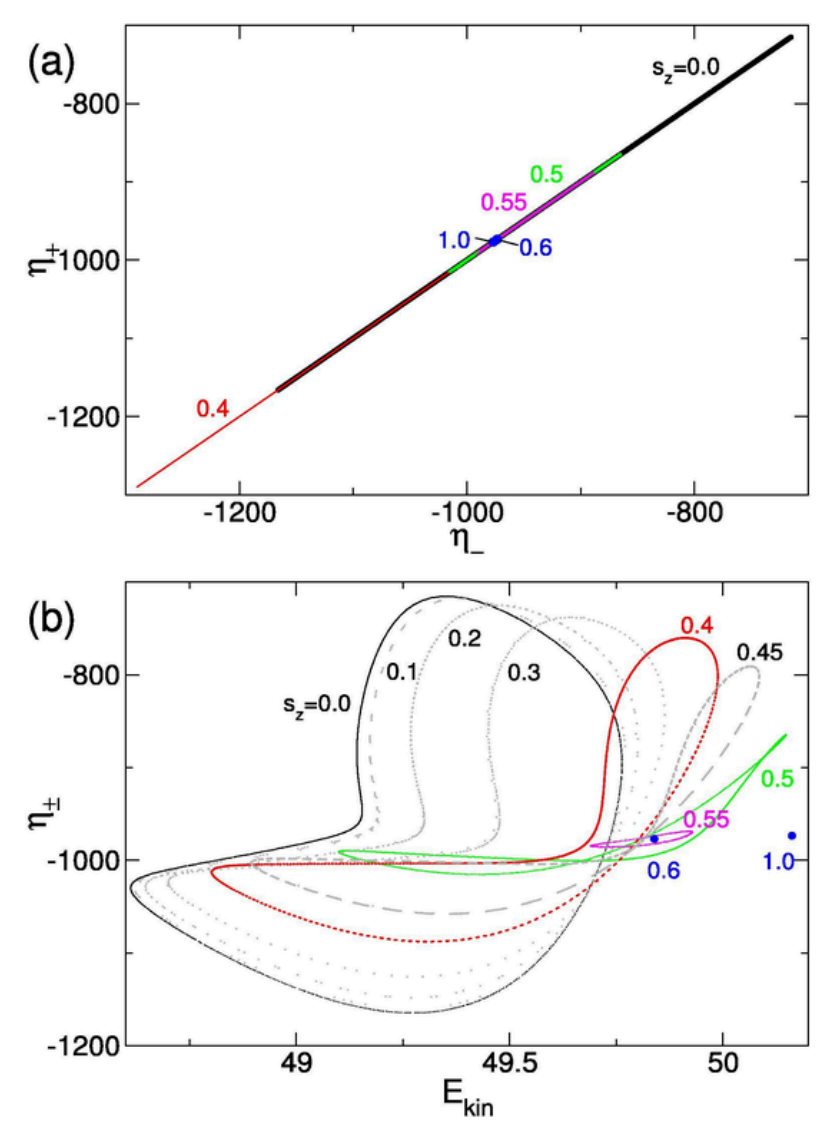

Fig. 6. Phase space for $s_{z}$. Phase portraits of $\mathrm{pV}^{\mathrm{s}}$ at different $s_{z}$ as indicated in $(a)\left(\eta_{-}, \eta_{+}\right)$ and $(b)\left(E_{k i n}, \eta_{ \pm}\right)$plane. Numbers in the figure identify the magnetic field strength $s_{z \text {. Due }}$ to symmetry reason, the limit cycle solutions appear due to projection in $\left(\eta_{-}, \eta_{+}\right)$, as a single line (degenerated solution) on the diagonal line $\eta_{-}=\eta_{+}(a)$, but can be clearly indicated in $(b)$. Same color code is used in both presentations $(a, b)$.

\subsection{Bifurcation scenario and period time for $s_{x} \neq 0$}

As before, we will start our discussion by looking at the corresponding bifurcation diagram shown in Fig. 8 illustrating the evolution of (time-averaged) modal kinetic energy $\bar{E}_{k i n}$ and corresponding time period $\tau$ with $s_{x}$.

Analog to the scenario for pure axial magnetic field, we start in the symmetric solution $\mathrm{pV}^{\mathrm{S}}$ in absence of any magnetic field. As soon as $s_{x} \neq 0$, the flow becomes inherently 3D due former mentioned reasons and thus the solution $\mathrm{pV}_{2}^{s}$ is present. $\mathrm{pV}_{2}^{s}$ features basically the same dynamics as $\mathrm{pV}^{\mathrm{s}}$. Increasing $s_{x}$ results in a monotonously growing kinetic energy $\bar{E}_{k i n}$ with an almost linear behavior/slope for $s_{x} \gtrsim 0.4$ before eventually the flow becomes stationary, $6 \mathrm{~V}_{2}$, at $s_{x} \approx 0.77$. The magnetic field induced 2-fold symmetry ( $m=2$ modes) which renders the solution to be $3 \mathrm{D}$ is clearly visible in Fig. 12 which presents different perspectives of $6 \mathrm{~V}_{2}$ at $s_{x}=0.775$. However, different to the scenario for axial magnetic field, in presence of transverse magnetic fields, $\mathrm{pV}_{2}^{s}$ does not move/end direct into the stationary solution $6 \mathrm{~V}_{2}$. Instead it loses it's stability at $s_{x} \approx 0.72$ in favor of another, coexisting propagating vortex state, $\mathrm{pV}_{2}^{a}$, which then finally disappears into $6 \mathrm{~V}_{2}$. Starting in the stationary solution $6 \mathrm{~V}_{2}$ and decreasing $s_{x}$ the corresponding sequence reads as follows: At the pitchfork bifurcation at $s_{x} \approx 0.77 \mathrm{pV}_{2}^{a(*)}$ appears as stable solution while $\mathrm{pV}_{s}^{s}$ only exists unstable. Decreasing $s_{x}, \mathrm{pV}_{2}^{a}$ undergoes a symmetry breaking Hopf bifurcation into another modulated solution $\mathrm{pV}_{2}^{a m}$ (see below), which finally loses stability at $s_{x} \approx 0.64$ to move transient into the only stable solution $\mathrm{pV}_{2}^{s}$. The latter remains stable existing for the whole parameter until $s_{x}$ disappear, ending in $\mathrm{pV}^{\mathrm{s}}$.

\subsubsection{Flow structures and transition scenarios with $s_{x}$}

The former mentioned transition scenario from $\mathrm{pV}_{2}^{s}$, losing it's stability and then changing towards the stable solution $\mathrm{pV}_{2}^{a}$ is illustrated in Fig. 9 for $s_{x}=0.71$. Although both, $\mathrm{pV}_{2}^{s}$ and $\mathrm{pV}_{2}^{a}$ describe solutions with propagating vortices, there are some key differences.

While $\mathrm{pV}_{2}^{s}$ fulfill the basic system symmetries in presence of a transverse magnetic field (Eq. (5)), for $\mathrm{pV}_{2}^{a}$ the basic $K_{z}^{H_{x}}$ symmetry is broken. Instead $\mathrm{pV}_{2}^{a}$ only has a complexer spacetime symmetry, which is donated/known as half-period flip $S^{H_{x}}$, where $K_{z}^{H_{x}}\left(p V_{2}^{a}(t)\right)=p V_{2}^{a}(t+\tau / 2)$. The effect of $S^{H_{x}}=K_{z}^{H_{x}} \Phi_{\tau / 2}^{H_{x}}$ on the velocity field is

$S^{H_{x}}\left(u, v, w, H_{x}\right) s(r, \theta, z, t)$

$=\left(u, v,-w,-H_{x}\right)(r, \theta,-z, t+\tau / 2)$.

However, it is worth to mention that this half-period-flip is slightly different from the common one featured in TCS, which usually includes/ involves a rotation of the flow structure. In the present scenario no azimuthal rotation is present. All propagation and motion dynamics exclusively appear in axial direction. The snapshots at $t=0(2 a)$ and $t=\tau / 2$ (2c) in Fig. 10 highlight the half-period-flip symmetry $S^{H_{x}}$ of $\mathrm{pV}_{2}^{a}$.

Fig. 9 shed some light into this change in symmetry from different perspectives. First of all the spacetime plots of $u(\theta, z)$ at mid-gap clearly show a change in the flow pattern. At the beginning of the transformation $\left(\mathrm{pV}_{2}^{s}\right)$ (Fig. $9(a)$ ) the black zero-contour lines as indicator for the propagating vortices, appear symmetrically near the upper and lower lids (as for $\mathrm{pV}^{\mathrm{s}}$ in Fig. 2) in a kind of elliptic pattern pointing towards
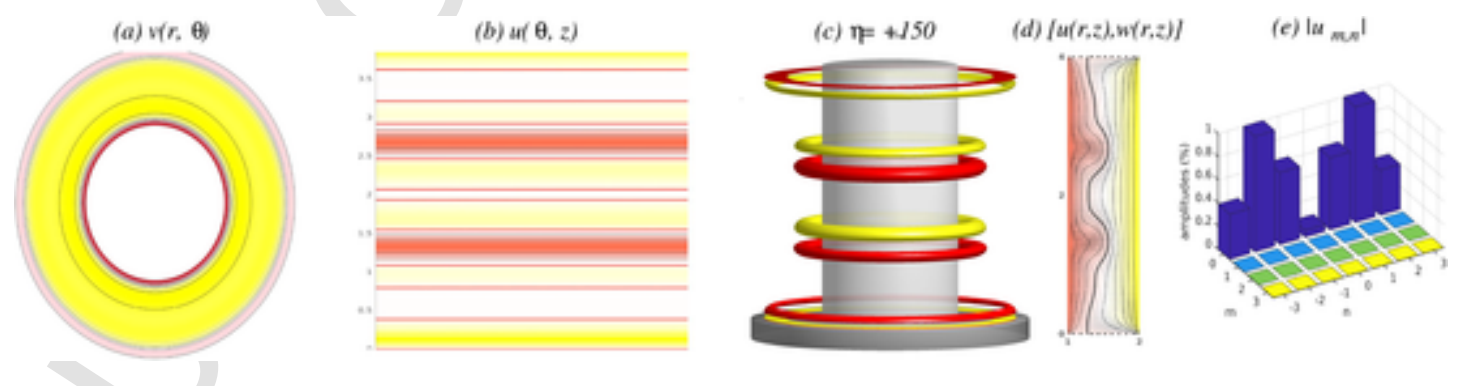

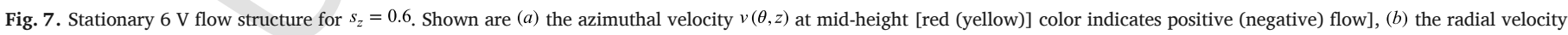

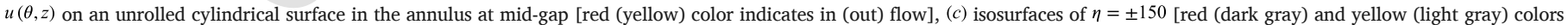

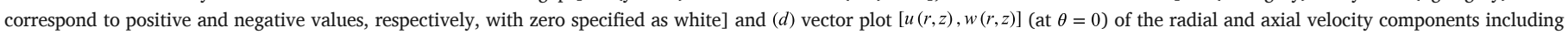

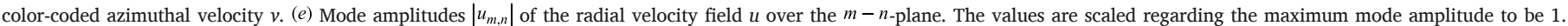

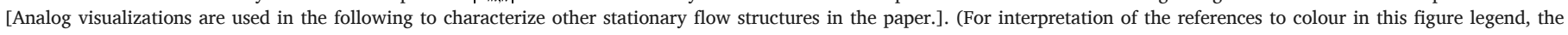
reader is referred to the web version of this article.) 


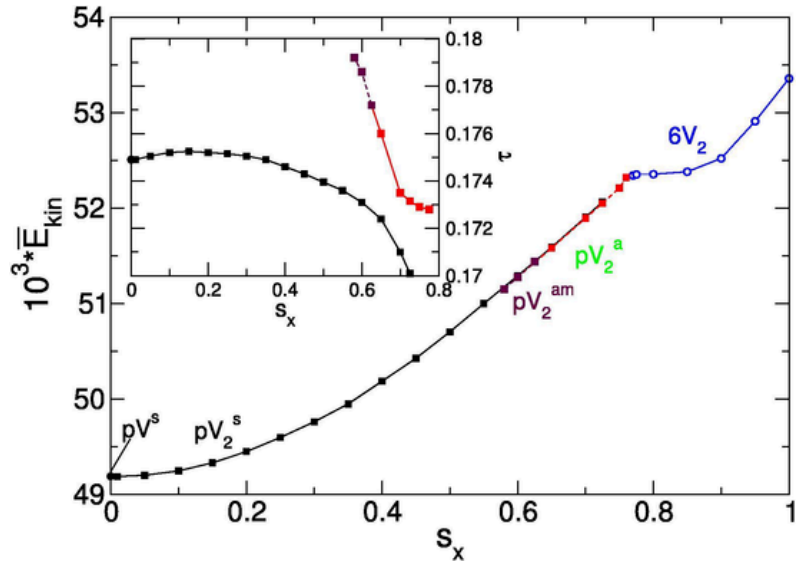

Fig. 8. Bifurcation diagram and period time evolution as Fig. 3 but for variation with $s_{x}$. Note that all flow structures are 3D except for $\mathrm{p} . \mathrm{V}^{s}$.

the mid-height region and to the right (time evolution). In contrast at the end of the transition scenario in $\left(\mathrm{pV}_{2}^{a}\right)($ Fig. $9(c))$ a pattern with alternate appearance between top and bottom of these elongated elliptic regions is present. Corresponding time evolution of the local measures $\eta_{+}$and $\eta_{-}$(Fig. $9(2 b, 2 c)$ ) during the transition show the initial synchronized motion in time for $\eta_{ \pm}$from which it moves toward a half period shifted time evolution for $\eta_{+}$and $\eta_{-}$, respectively. In the similar manner the phase portrait $\left(\eta_{+}, \eta_{-}\right)$elucidate the evolution from the symmetric $\mathrm{pV}_{2}^{s}$, degenerated limit cycle solution, appearing on the diagonal with $\eta_{+}=\eta_{-}$towards the asymmetric $\mathrm{pV}_{2}^{a}$ state, exploring a wider region in $\left(\eta_{+}, \eta_{-}\right)$parameter space. Note that $\mathrm{pV}_{2}^{a}$ remains symmetric via mirroring at the diagonal $\eta_{+}=\eta_{-}$.

For a direct comparison of these different types of propagating vortex states, both Figs. 10 and 11 illustrate different perspectives and features of $\mathrm{pV}_{2}^{s}$ and $\mathrm{pV}_{2}^{a}$, which are coexisting at $s_{x}=0.65$. Fig. 10 shows flow visualizations over one period while Fig. 11 gives a more quantitative measure with the PSD of $E_{k i n}, \eta_{ \pm}$aside spacetime plots. For a better impression of the dynamics see also the movies movie_pVs2_sx065.avi and movie_pVa2_sx065.avi in SM.

Regarding Fig. 10, both, 3D plots of the azimuthal vorticity, $\eta$, and the radial velocity $u(r, \theta)$ at cylinder mid-gap indicate the either symmetrically motion (from lids towards mid-height) of vortices for $\mathrm{pV}_{2}^{s}$ or the alternate appearance and motion of these vortices for $\mathrm{pV}_{2}^{a}$. Aside, the 2-fold symmetry ( $m=2$-mode) stimulated by the symmetry breaking transverse magnetic field is clearly visible in $u(r, \theta)$ for $\mathrm{pV}_{2}^{s}$ and $\mathrm{pV}_{2}^{a}$ , respectively (Fig. 10). This also held for the stationary state $6 \mathrm{~V}_{2}$ (Fig. $12(b))$.

While the averaged kinetic energy $\bar{E}_{k i n}$ for $\mathrm{pV}_{2}^{s}$ and $\mathrm{pV}_{2}^{a}$ are virtually indistinguishable and fall together the corresponding period times $\tau$ are slightly different (insight in Fig. 8). Regarding $\mathrm{pV}_{2}^{s}$, the trend is similar to the scenario under axial magnetic fields, with the only difference, that the period time $\tau$ is not fully monotonously. Here $\tau$ first
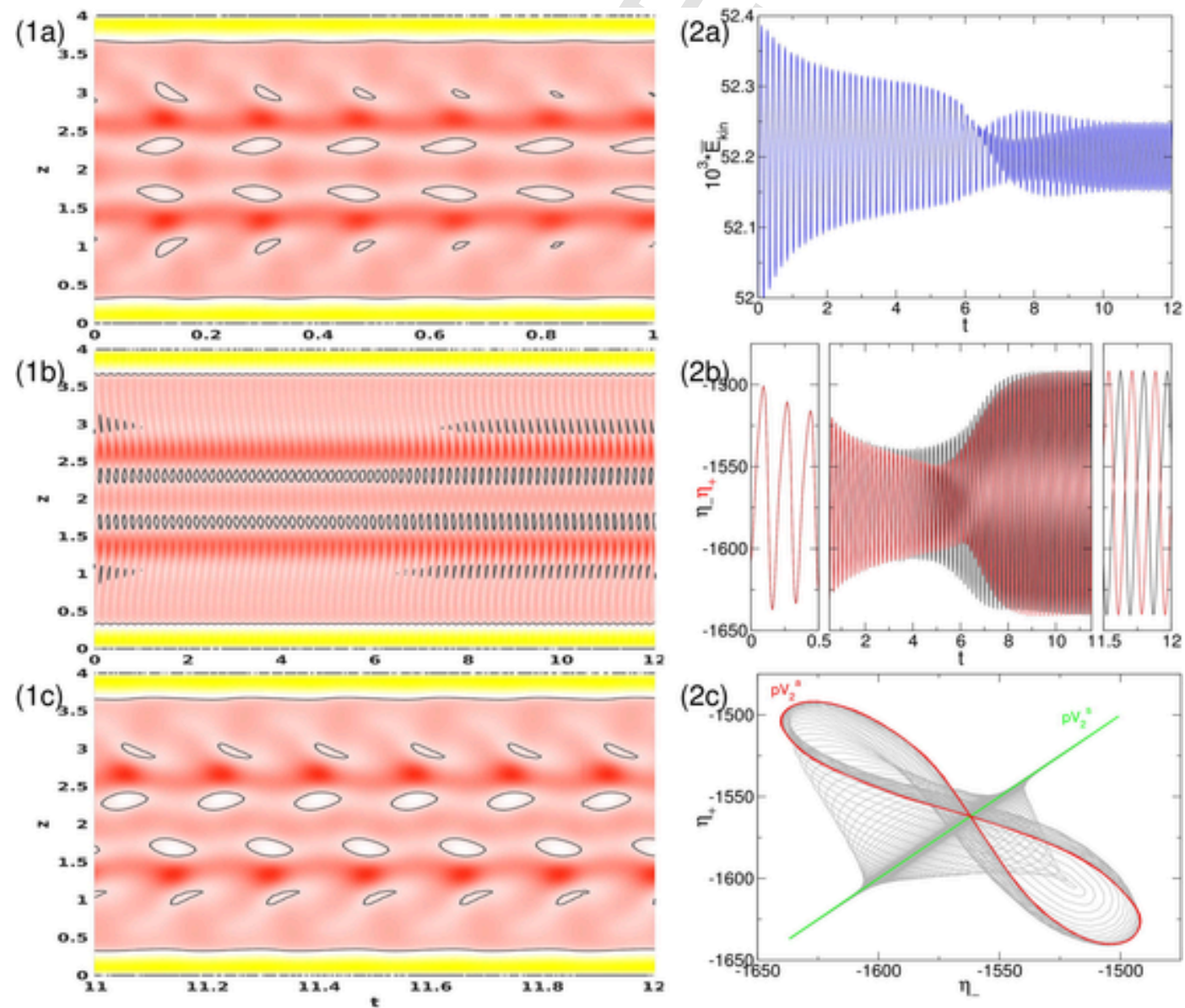

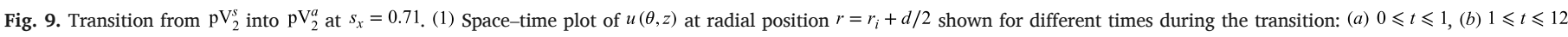

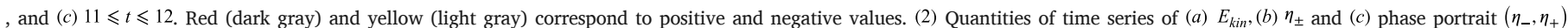

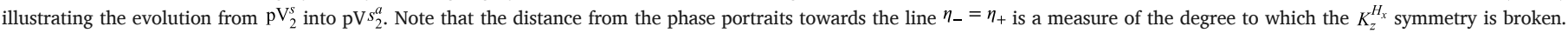
(For interpretation of the references to colour in this figure legend, the reader is referred to the web version of this article.) 

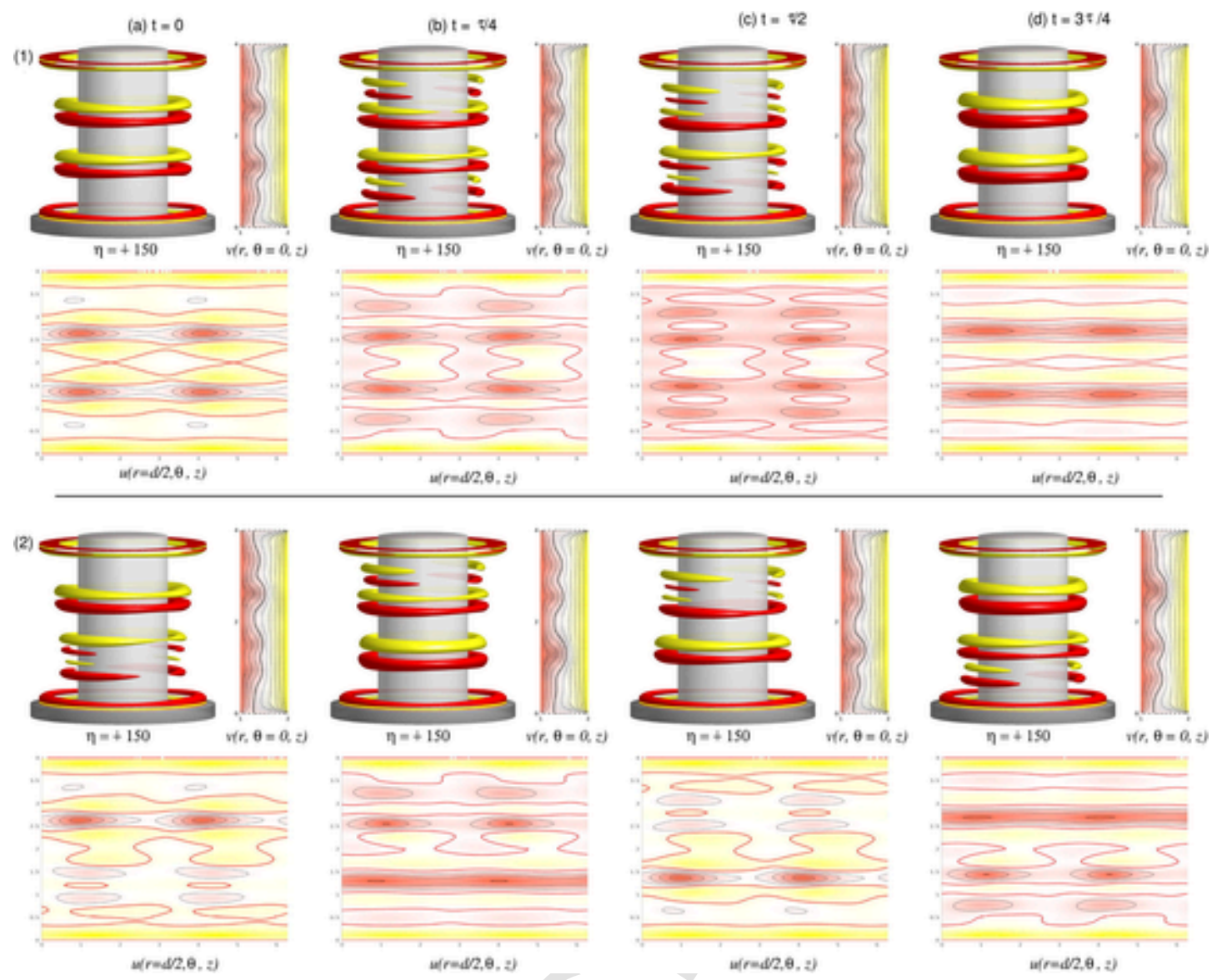

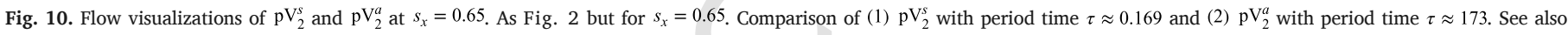
movie_pVs2_sx065.avi and movie_pVa2_sx065.avi in SM. Note the snapshots at $t=0(2 a)$ and $t=\tau / 2(2 c)$ illustrate the half-period-flip symmetry $S^{H_{x}}$ of pV ${ }_{2}^{a}$.

slightly increase, before it decreases with $s_{x}$. While similar decrease in $\tau$ for $\mathrm{pV}_{2}^{a}$ and $\mathrm{pV}_{2}^{a m}$ is visible, there is an obvious significant smaller variation in $\tau$ close to the onset.

Fig. 12 illustrates flow visualizations of the stationary $6 \mathrm{~V}_{2}$ flow structures at $s_{x}=0.775$ out of which both, $\mathrm{pV}_{2}^{s}$ and $\mathrm{pV}_{2}^{a(*)}$ bifurcate from in a pitchfork bifurcation (at $s_{x} \approx 0.77$ ) with decreasing $s_{x}$. However, while $\mathrm{pV}_{2}^{a}$ appears out of $6 \mathrm{~V}_{2}$ as stable solution, $\mathrm{pV}_{2}^{s}$ is unstable close to the onset and becomes stabilized at $s_{x} \approx 0.72$ and hereafter remains stable towards the point of vanishing magnetic field $s_{x}=0$. Either mode amplitudes $\left|u_{m, n}\right|$ and radial flow $u(r, \theta)$ (Fig. 12(b,e)) highlight the stimulated $m=2$ modes due transversal magnetic field.

The evolution of the limit cycle solution $\mathrm{pV}_{2}^{a}$ (at $s_{x}=0.76$, i.e. close to onset) out of the fixed point solution $6 \mathrm{~V}_{2}$ is illustrated in Fig. 13. Starting with a single point in phase space (Fig. 13(2c)) the flow becomes time dependent and the dynamic changes into the limit cycle solution $\mathrm{pV}_{2}^{a}$ with alternating motion in upper and lower system half, as can be detected in time series of $\eta_{ \pm}$as well the spacetime plot (Fig. 13 $(1,2 b))$.

Fig. 14 shows corresponding power spectral densities (PSDs) and time series of $E_{k i n}$ and $\eta_{ \pm}$for $\mathrm{pV}_{2}^{a}$ close to onset at $s_{x}=0.76$. Note that here $\tau$ is twice the period of the time series of $E_{k i n}$; this is because $\mathrm{pV}_{2}^{a}$ is half-period-flip invariant and so $E_{k i n}\left(p V_{2}^{a}(t)\right)=E_{k i n}\left(p V_{2}^{a}(t+\tau / 2)\right)$ , whereas $\mathrm{pV}_{2}^{a}$ is $\tau$ periodic, $p V_{2}^{a}(t)=p V_{2}^{a}(t+\tau)$.

It is worth to mention that at the bifurcation point, only $\mathrm{pV}_{2}^{a}$ bifurcates stable out of the stationary solution $6 \mathrm{~V}_{2}$, while $\mathrm{pV}_{2}^{s}$ is unstable close to onset. Applying symmetry restriction to our numerical simulation we could also observe the unstable symmetric $\mathrm{pV}_{2}^{s}$ state close to onset. However, releasing the restrictions the solution always moves towards the asymmetric $\mathrm{pV}_{2}^{a}$ state. But worth to mention as this depends on various system parameters, one cannot exclude that for another setup also symmetric $\mathrm{pV}_{2}^{s}$ states may bifurcate primary and stable, as it does for pure axial field configuration (cf. Fig. 8). At some distance apart of the onset $\mathrm{pV}_{2}^{s}$ becomes stabilized and thus coexisting with $\mathrm{pV}_{2}^{a}$.

After being stabilized, with decreasing $s_{x}$ the flow $\mathrm{pV}_{2}^{s}$ continuous to exist with minor changes in it's dynamics until $s_{x}=0$. This scenario is very similar to the one discussed before for axial magnetic field. Basically only the period time $\tau$ changes slightly. However, the scenario for the asymmetric solution $\mathrm{pV}_{2}^{a}$ becomes more complicate. At $s_{x} \approx 0.64$ $\mathrm{pV}_{2}^{a}$ undergoes a symmetry breaking Hopf bifurcation. Here the half-period-flip symmetry $S^{H_{x}}$ becomes broken. Following this, both periodic solutions evolve on one or other symmetrically related limit cycles $\mathrm{pV}_{2}^{m a}$ and $p V_{2}^{m a *}=K_{z}^{H_{x}} p V_{2}^{m a}$ (the index $m$ indicate the modulation).

\subsubsection{Phase Space and asymmetry characteristics with $s_{x}$}

Visually (by pure eye) $\mathrm{pV}_{2}^{a}$ and its modulated cousin, $\mathrm{pV}_{2}^{a m}$, are indistinguishable (see also Fig. 2 in SM). However, either in the time series of $\eta_{ \pm}$and in the phase space projection the broken half-period-flip symmetry $S^{H_{x}}$ is observable (Fig. 15(b)).

Fig. 15(a) provides time series of $\eta_{+}, \eta_{-}, \eta_{+}(t+\tau / 2)\left(\eta_{+}\right.$with half period shift $\tau / 2)$ and the difference $\Delta \eta(t, t+\tau / 2)=\eta_{+}(t)-\eta_{-}(t+\tau / 2)$. Although by pure eye hardly any variation between $\eta_{+}$and $\eta_{+}\left(t+\tau_{2}\right)$ is observable, $\Delta \eta$ clearly reveals the differences. $\Delta \eta=0$ for $p V_{2}^{a}$ at $s_{x}=0.65$ and having finite values $\Delta \eta \neq 0$ for $p V_{2}^{a m}$ at $s_{x}=0.6$ shows the change from being half-period-flip symmetric to a broken half-period flip symmetry. The phase space projection (Fig. 15(b)) illustrates 

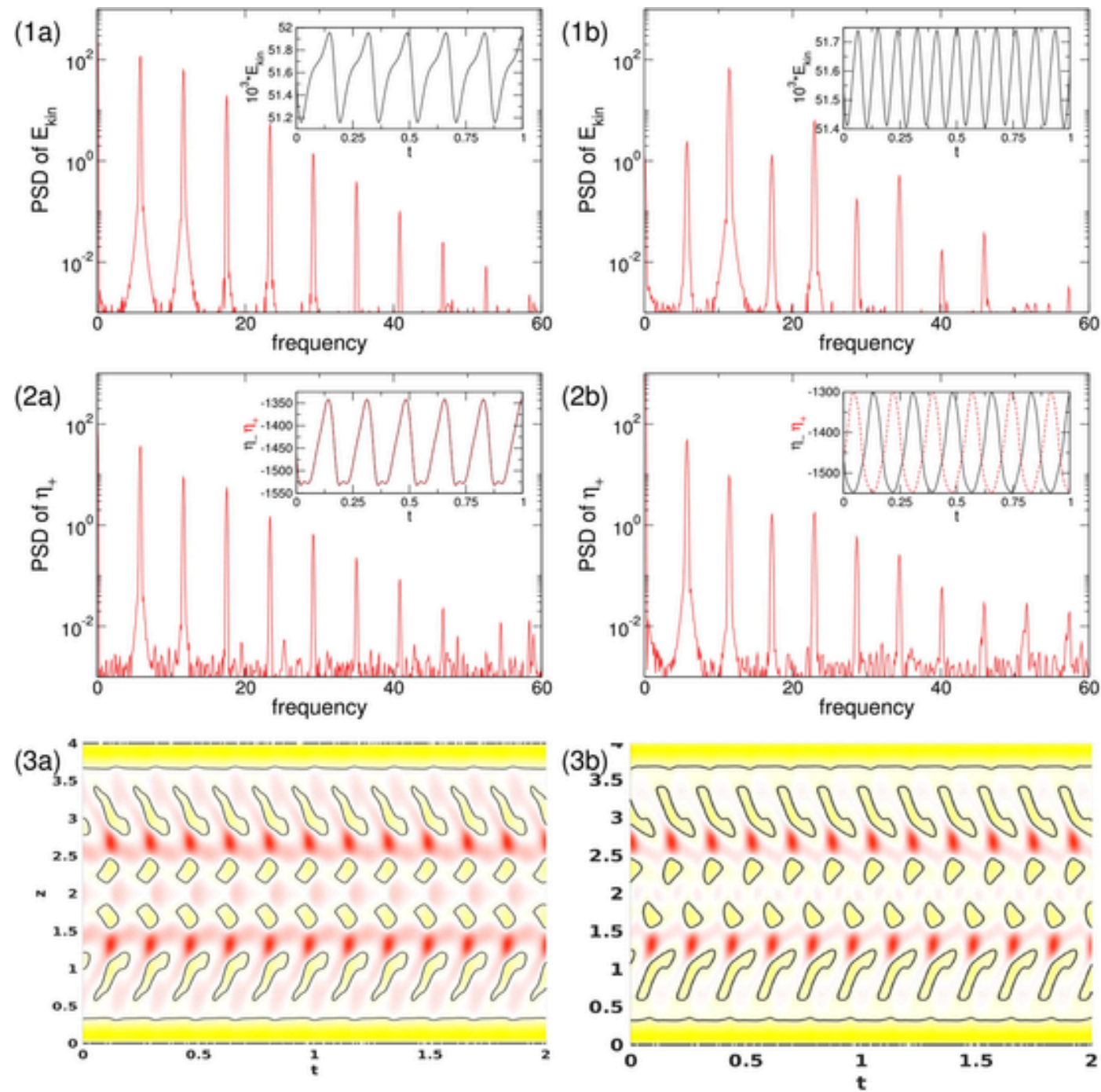

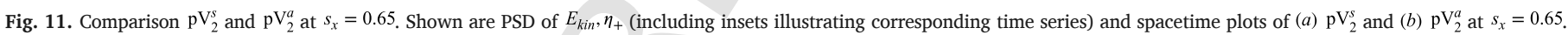
See also movie_pVs2_sx065.avi and movie_pVa2_sx065.avi in SM.
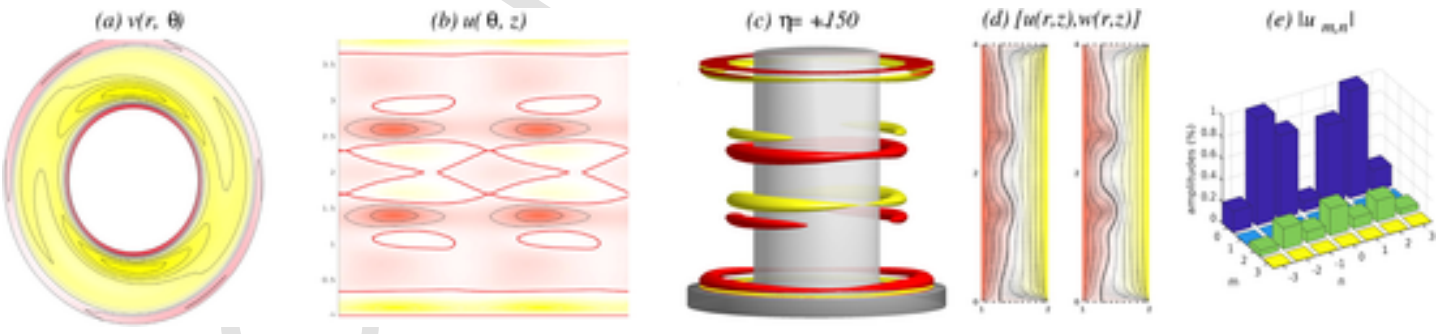

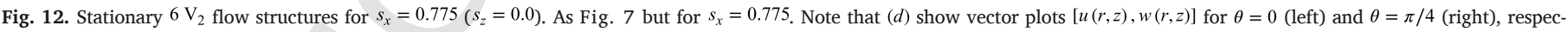
tively, illustrating the 3D characteristics due to $m=2$ mo.de stimulation.

this behavior with broken $S^{H_{x}}$ symmetry for $p V_{2}^{a m}$. While $\mathrm{pV}_{2}^{a}$ for $s_{x}=0.65$ appears symmetric with respect to the diagonal line $\eta_{-}=\eta_{+}$ this symmetry is lost for $\mathrm{pV}_{2}^{a m}$ at $s_{x}=0.6$ (cf. Fig. 16).

Fig. 16 shows phase portraits, both in $\left(\eta_{+}, \eta_{-}\right)$and $\left(\eta_{+}, E_{k i n}\right)$ planes representing all flow structures with variation in $s_{x}$. As already seen for axial magnetic field the $K_{z}^{H_{x}}$ symmetric solutions $\mathrm{pV}_{2}^{s}$ appear as degenerated limit cycles to lie on the diagonal $\eta_{-}=\eta_{+}$. But note, in contrast to pure axial field, here $\mathrm{pV}_{2}^{s}$ are fully 3D. $\mathrm{pV}_{2}^{a}$ appear reflection symmetry with respect to the diagonal $\eta_{-}=\eta_{+}$, which finally disappear for $\mathrm{pV}_{2}^{a m}$, existing as two degenerated solutions.
In order to further quantitative measure the asymmetry between the different flow structures $\mathrm{pV}_{2}^{s}, \mathrm{pV}_{2}^{a}$, and $\mathrm{pV}_{2}^{a m}$, we defined an asymmetry parameter as $\eta_{A}=\left(\Delta \eta_{+}{ }^{2}-\Delta \eta_{-}^{2}\right)^{1 / 2}$ with $\Delta \eta_{ \pm}=\max \left(\eta_{ \pm}\right)-\min \left(\eta_{ \pm}\right)$ . By this $\eta_{A}$ measures the asymmetry regarding the diagonal $\eta_{+}=\eta_{-}$ . The variation with $s_{x}$ for $\eta_{A}$ is shown in Fig. 17. As a result, that $\mathrm{pV}, 6 \mathrm{~V}_{2}$ and $\mathrm{pV}_{2}^{s}$ all fulfill the basic system reflection symmetry $K_{z}^{H_{x}}$ (i.e $K_{z}$ for $\mathrm{pV}$ in absence of a magnetic field) $\eta_{A}=0$ for all these states. On the other hand the broken $K_{z}^{H_{x}}$ symmetry, replaced by the half period-flip-symmetry, $S^{H_{x}}$, results in a continuously increasing magnitude/ampli- 

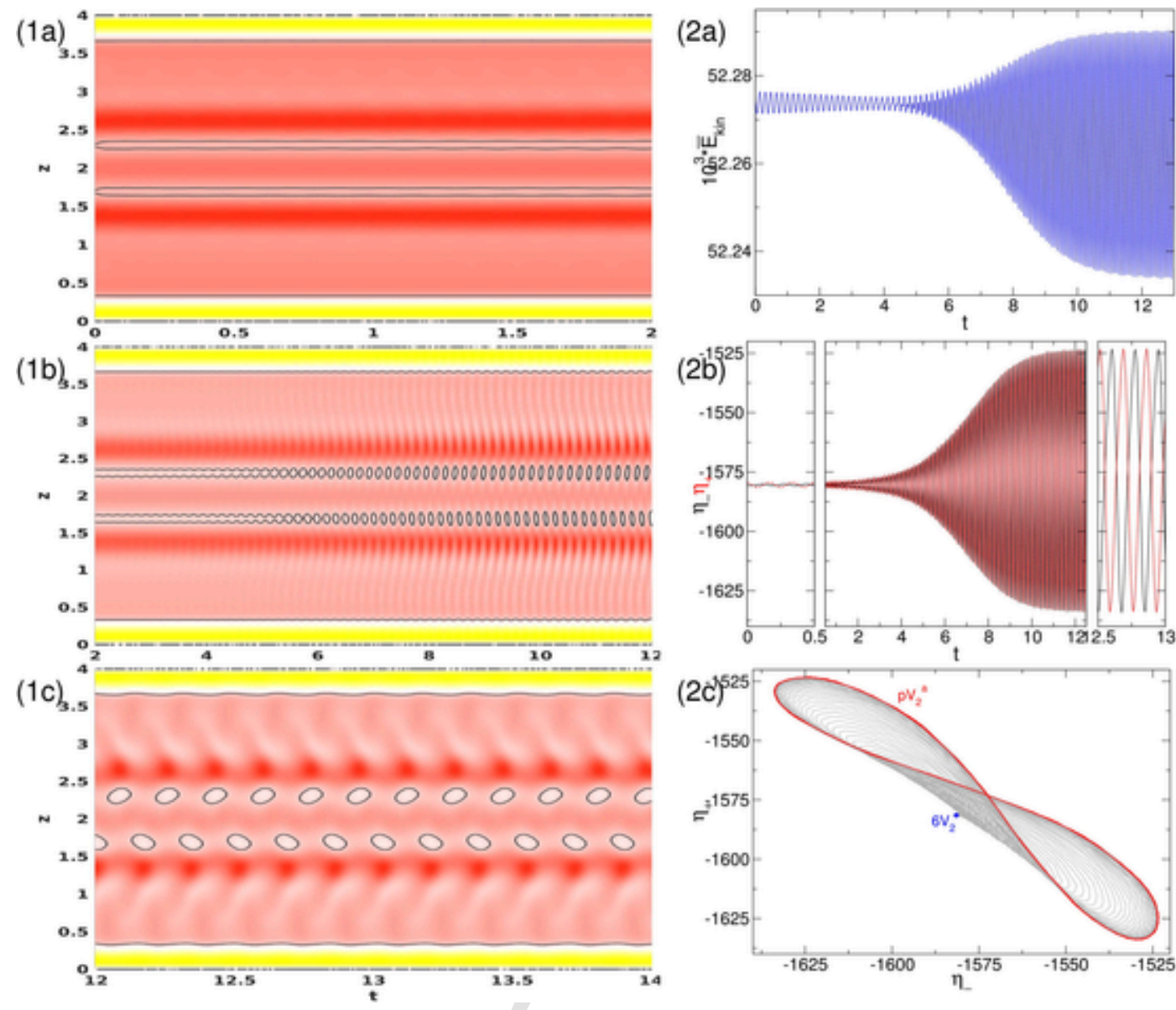

Fig. 13. Evolution from $6 \mathrm{~V}_{2}$ into $\mathrm{pV}_{2}^{a}$ at $s_{x}=0.76$. As Fig. 9, but for the transient evolution from $6 \mathrm{~V}_{2}$ into $\mathrm{pV}_{2}^{a}$ at $s_{x}=0.76$.

tude $\eta_{A}$ from the onset at $s_{x} \approx 0.77$. Thus the solutions become more asymmetric, also visible in the continuously exploration of a wider region in phase space in $\left(\eta_{+}, \eta_{-}\right)$plane with decreasing $s_{x}$. This trend even increase with the loss of $S^{H_{x}}$ and the appearance of the two symmetry related limit cycles $\mathrm{pV}_{2}^{a m}$ and $\mathrm{pV}_{2}^{a m *}$ at $s_{x} \approx 0.63$ (Fig. 15).

\section{Discussion and conclusion}

This paper is the first of two parts dealing with propagating vortices (pVs) in ferrofluidic Couette flow under influence of external magnetic fields. In the current manuscript only pure magnetic fields, either pure axial or pure transversal (i.e. symmetry breaking) orientated are considered. In particular for the latter the numerical simulations of the small-aspect-ratio wide-gap counter-rotating system have revealed a sequence of pitchfork and symmetry-breaking Hopf bifurcations as the magnetic field strength $s_{x}$ is changed. While in earlier studies in classical TCS and absence of any magnetic fields also asymmetric $\mathrm{pV}^{a}$ has been observed as stable solutions $[29,39]$, at the bifurcation point the symmetric $\mathrm{pV}^{\mathrm{s}}$ were detected to evolve primary stable out of $\mathrm{nV}$ states. The same holds for pure axial magnetic field. The presence of a symmetry breaking transverse magnetic field crucially influence and modify this characteristic in favor of a stable asymmetric state, $\mathrm{pV}_{2}^{a}$, at least close to onset.

A schematic of the bifurcation sequences under transversal magnetic field, as discussed above, using $s_{x}$ as bifurcation parameter (decreasing from left to right), is shown in Fig. 18. First the base state $\mathrm{CCF}_{2}$ disappear with the evolution of sub-critical vortices [29] forming the steady fixed point solution $6 \mathrm{~V}_{2}$. Both $\mathrm{CCF}_{2}$ and $6 \mathrm{~V}_{2}$ are invariant under the full system symmetry of the problem under transverse magnetic field,
$S O(2)^{H_{x}} \times Z_{2}^{H_{x}} \times \mathbb{R}$. At $P$, the stationary state, $6 \mathrm{~V}_{2}$, undergoes a pitchfork bifurcation resulting in the stable limit cycle solution, $\mathrm{pV}_{2}^{a(*)}$, as well as the unstable limit cycle solution $\mathrm{pV}_{2}^{s} \cdot \mathrm{pV}_{2}^{a(*)}$ brakes the $K_{z}^{H_{x}}$ symmetry and the time invariance $\Phi$ of $6 \mathrm{~V}_{2}$. The limit cycle, however, is invariant to a combination of the two broken symmetries, consisting of $K_{z}^{H_{x}}$ composed with a half-period time translation $S^{H_{x}}$. This half-period-flip symmetry is then in the following broken in the Hopf bifurcation $\mathrm{H}$ and two limit cycle solutions $\mathrm{pV}_{2}^{a m}$ and $\mathrm{pV}_{2}^{a m *}$ result. These are symmetrically related: $p V_{2}^{a m, *}=K_{z}^{H_{x}} p V_{2}^{a m}$. Eventually $\mathrm{pV}_{2}^{a m, *}$ lose stability and move transient to the only remaining stable solution $\mathrm{pV}_{2}^{s}$, which already appeared in $\mathrm{P}$ but being unstable close to onset.

The main main/key features and results regarding propagating vortices (pVs) under pure axial or pure transversal magnetic field in short aspect TCS can be summarized as follows:

\section{Axial magnetic field}

- All propagating vortices $\left(\mathrm{pV}^{\mathrm{s}}\right)$ remain $2 \mathrm{D}$.

- All propagating vortex structures, $\mathrm{pV}^{\mathrm{s}}$, always feature same flow dynamics and crucially identical symmetry

$K_{z}$ (no stable asymmetric $\mathrm{pVs}$ could be found).

The first point is true in general, also including modified boundary conditions [30]. However, the second point only applies for finite systems with fixed lids at bottom and top. Any change to different boundary conditions in axial direction immediately destroys the $K_{z}$ symmetry. On top of this other system parameters may also play a role. 

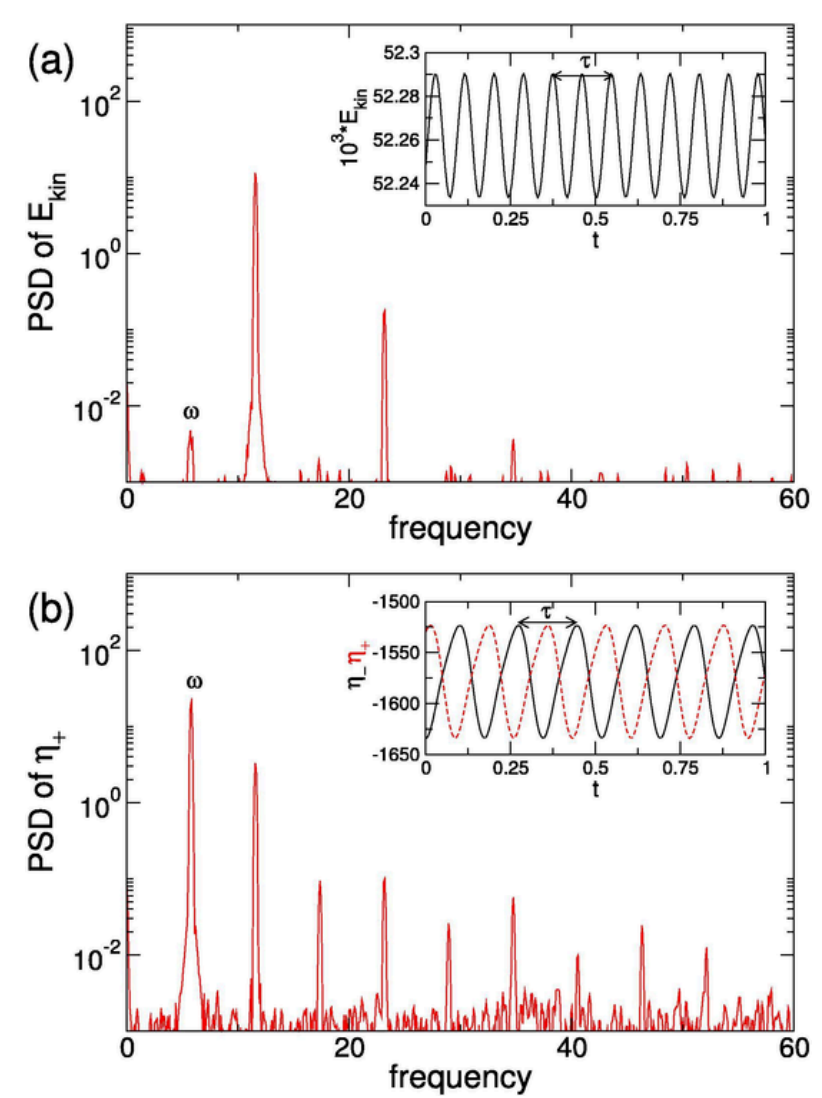

Fig. 14. Time series and PSDs of $\mathrm{pV}_{2}^{a}$ at $s_{x}=0.76$. PSDs of $(a) E_{k i n}$ and $(b) \eta_{+}$for the asymmetric propagating flow state $\mathrm{pV}_{2}^{a}$ close to onset at $s_{x}=0.76$. Period time $\tau \approx 0.171$ with corresponding frequency $\omega \approx 5.853$. Insets show time series of $E_{k i n}, \eta_{+}$[red], $\eta_{-}$[black].

\section{Transverse magnetic field}

- All propagating vortices (and flow structures $[13,14]$ ) are inherentially 3D.

- Different propagating vortex structures can appear coexisting, featuring different symmetries:

(i) $\mathrm{pV}_{2}^{s}$, with basic system symmetry, $K_{z}^{H_{x}}$, under transversal magnetic field.

(ii) $\mathrm{pV}_{2}^{a}$, with half-period-flip symmetry $S^{H_{x}}$.

(iii) $\mathrm{pV}_{2}^{a m}$, without discrete symmetry, but existing as pair of asymmetric solutions $\mathrm{pV}_{2}^{a m}$ and $p V_{2}^{a m, *}=K_{z}^{H_{x}} p V_{2}^{a m}$,

- A symmetry breaking transverse magnetic field triggers the asymmetric solution $\mathrm{pV}_{2}^{a}$ to be stable at it's bifurcation point, while $\mathrm{pV}_{2}^{s}$ is unstable close to onset.

- $\mathrm{pV}_{2}^{s}$ and $\mathrm{pV}_{2}^{a}\left[\mathrm{pV}_{2}^{a m}\right]$ are coexisting over certain parameter range $s_{x}$.

Important to emphasis, all here studied propagating vortices, either under axial or transversal magnetic field condition, are topological speaking represented by relative simple limit cycle (1-torus) solutions. Neither the fact of symmetric $\left(\mathrm{pV}^{\mathrm{s}}\right)$, alternate (asymmetric) appearing vortices $\left(\mathrm{pV}^{a}\right)$, or even modulation of the latter changes this topology.

In the second part of this study we focus on the dynamics and behavior of propagating vortices under the influence of an oblique magnetic field [34] as superposition of axial and transversal magnetic field. Such superposition of fields not only result in further non-linear interaction and complexer flow solutions it also crucially modifies the basic
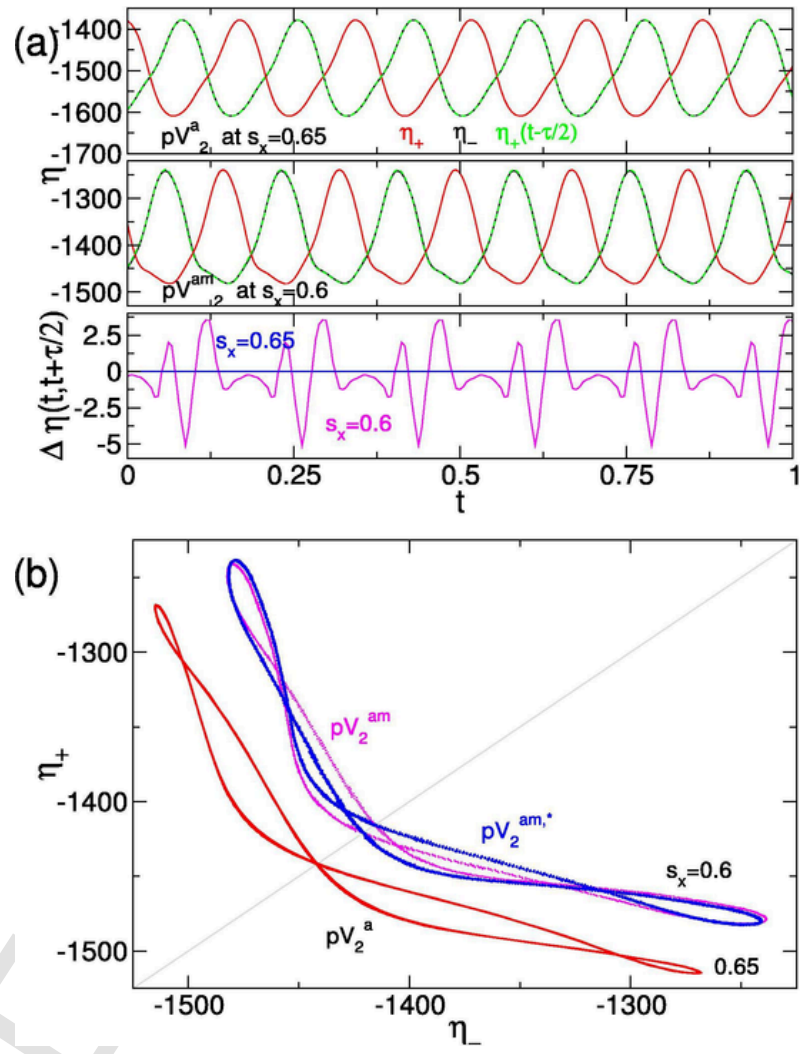

Fig. 15. Comparison between $\mathrm{pV}_{2}^{a}$ and $\mathrm{pV}_{2}^{a m}$. (a) Variation and differences in $\eta_{ \pm}$for $\mathrm{pV}_{2}^{a}$ at $s_{x}=0.65$ and $\mathrm{pV}_{2}^{a m}$ at $s_{x}=0.6$ before and after the half-period-flip-symmetry is broken, respectively. $\eta_{+}(t+\tau / 2)$ is $\eta_{+}$shifted by half a period $\tau / 2$. (b) Extraction of Fig. 16 showing the phase portrait in $\left(\eta_{-}, \eta_{+}\right)$around the appearance of the two symmetry related states $\mathrm{pV}_{2}^{a m}$ and $\mathrm{pV}_{2}^{a m *}$ (cf. Fig. 2 in SM for further visualizations and characteristics of $\mathrm{pV}_{2}^{a}$ and $\mathrm{pV}_{2}^{a m}$.

symmetries and more important alters the topology of the solutions. Therefore this scenario will be discussed separately in [34].

Despite that the here presented results are in good agreement with experimental findings, it is worth to point out that any effects of natural convection have been neglected. Especially in view of the recent increase in the number of non-isothermal situations wherein magnetic fluid are put to use in place of classical fluids, natural convection will play a role and can significant the flow dynamics. Although for given system parameters (basically isothermal conditions) such effects seems to be small, further experimental and numerical studies will have to be undertaken to investigate the influence of natural convection in ferrofluids in the presence of magnetic fields.

\section{CRediT authorship contribution statement}

Sebastian Altmeyer: Conceptualization, Methodology, Software, Data curation, Writing - original draft, Visualization, Investigation, Software, Validation, Writing - review \& editing.

\section{Declaration of Competing Interest}

The authors declare that they have no known competing financial interests or personal relationships that could have appeared to influence the work reported in this paper.

\section{Acknowledgments}

S. A. is a Serra Húnter Fellow. 

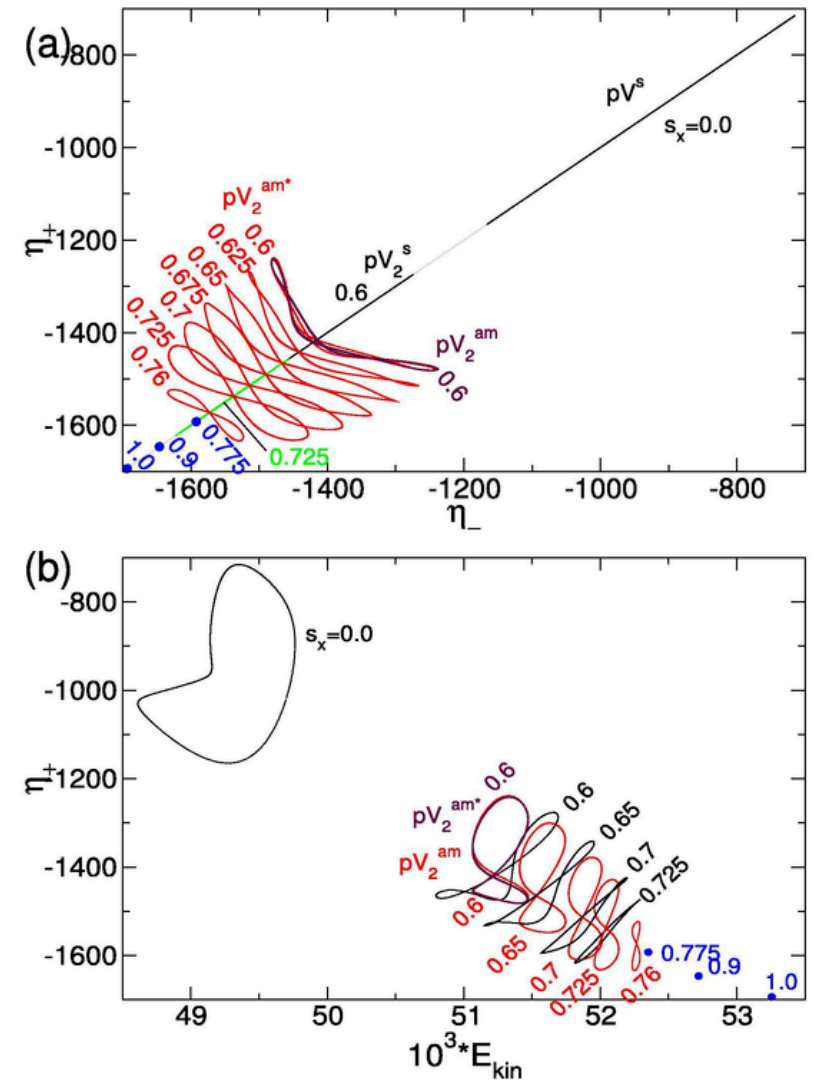

Fig. 16. Phase space projection for $s_{x} \neq 0$. Phase portraits of $\mathrm{pV}^{\mathrm{s}}, \mathrm{pV}_{2}^{a}$, and $\mathrm{pV}_{2}^{a m}$ for $s_{x}$ as indicated on $(a)\left(\eta_{-}, \eta_{+}\right)$and $(b)\left(E_{k i n}, \eta_{+}\right)$. Numbers in the figure identify the magnetic field strength $s_{x}$ as indicated (cf. Fig. 6).

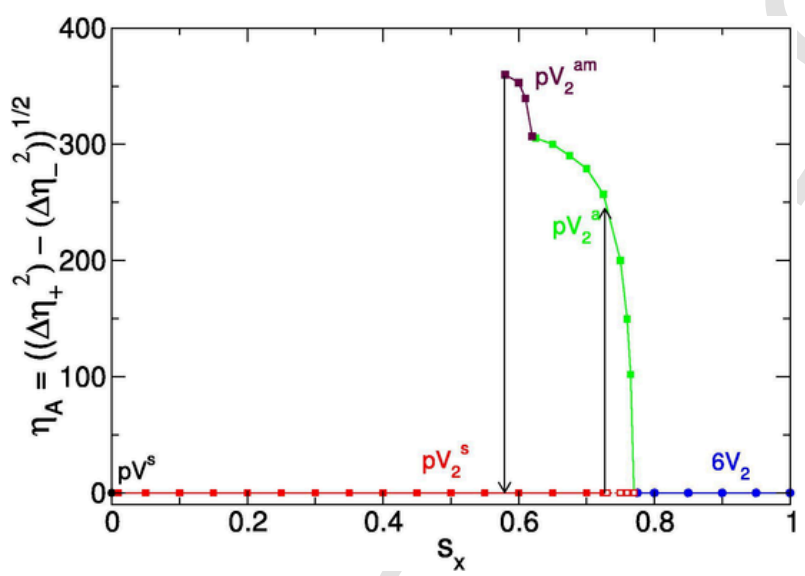

Fig. 17. Variation of $\eta_{A}$ with $s_{x}$ for pVs. Evolution of the asymmetry parameter $\eta_{A}$ with $s_{x}$ for different propagating vortex structures, $\mathrm{pV}^{\mathrm{s}}, \mathrm{pV}_{2}^{s}, \mathrm{pV}_{2}^{a}, \mathrm{pV}_{2}^{a m}$ and $6 \mathrm{~V}_{2}$. The vertical arrows indicate the transition scenario when one solution loses stability and the flow moves transient towards another stable solution.

\section{Appendix A.}

\section{A.1. Ferrohydrodynamical equation}

Eq. (1) is to be solved together with an equation that describes the magnetization of the ferrofluid. Using the equilibrium magnetization of an unperturbed state in which the homogeneously magnetized ferrofluid is at rest and the mean magnetic moment is orientated in the direction of the magnetic field, we have $\mathbf{M}^{\mathrm{eq}}=\chi \mathbf{H}$. The magnetic susceptibility $\chi$ of the ferrofluid can be approximated by the Langevin's for-

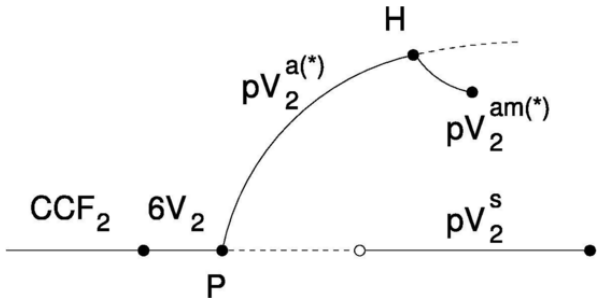

Fig. 18. Schematic bifurcation diagram, using $s_{x}$ as the bifurcation parameter. Here first the stationary $6 \mathrm{~V}_{2}$ state appears in a smooth transition out of the basic state $\mathrm{CCF}_{2}$. $\mathrm{P}$ denotes the pitchfork bifurcation $6 \mathrm{~V}_{2}$ into two stable, symmetry related flow states $\mathrm{p}$ $V_{2}^{a *}=K_{z}^{H_{x}} p V_{2}^{a}$ (stable) as well the $\mathrm{pV}_{2}^{s}$ (unstable), keeping the basic symmetries. All $\mathrm{pV}$ states are periodic and topological describe a limit cycle solution. Further a symmetry breaking Hopf bifurcation $\mathrm{H}$ appears, in which the half-period-flip symmetry $S^{H_{x}}$ of $\mathrm{pV}_{2}^{a}$ is broken, leading to pair of asymmetric solutions $\mathrm{pV}_{2}^{a m}$ and $p V_{2}^{a m *}=K_{z}^{H_{x}} p V_{2}^{a m}$, which eventually lose stability in favor of the only stable symmetric solution $\mathrm{pV}_{2}^{S}$. Stable (unstable) solution branches are shown as solid (dashed) curves.

mula [36], where we set the initial value of $\chi$ to be 0.9 and use a linear magnetization law. The ferrofluid studied corresponds to APG933 [41]. We consider the near equilibrium approximations of Niklas [8,27] (derived from the theory by Shliomis [6] under the assumption of a stationary magnetization) with a small value of $\left\|\mathbf{M}-\mathbf{M}^{\mathrm{eq}}\right\|$ and small magnetic relaxation time $\tau:|\nabla \times \mathbf{u}| \tau \ll 1$. Using these approximations, one can obtain [17] the following magnetization equation determining the relationship between the magnetization $\mathbf{M}$, the magnetic field $\mathbf{H}$, and the velocity $\mathbf{u}$

$\mathbf{M}-\mathbf{M}^{\mathrm{eq}}=c_{N}^{2}\left(\frac{1}{2} \nabla \times \mathbf{u} \times \mathbf{H}+\lambda_{2} \mathbb{S H}\right)$,

where

$c_{N}^{2}=\tau /\left(1 / \chi+\tau \mu_{0} H^{2} / 6 \mu \Phi\right)$

is the Niklas coefficient [8], $\mu$ is the dynamic viscosity, $\Phi$ is the volume fraction of the magnetic material, $\mathbb{S}$ is the symmetric component of the velocity gradient tensor $[17,35]$, and $\lambda_{2}$ is the material-dependent transport coefficient [42] which can be conveniently chosen to be $\lambda_{2}=4 / 5$ $[13,14,35,42]$. Using Eq. (6), we eliminate the magnetization from Eq. (1) to arrive at the following ferrohydrodynamical equations $[17,35]$ :

$\left(\partial_{t}+\mathbf{u} \cdot \nabla\right) \mathbf{u}-\nabla^{2} \mathbf{u}+\nabla p_{M}$

$=-\frac{s_{N}^{2}}{2}\left[\mathbf{H} \nabla \cdot\left(\mathbf{F}+\frac{4}{5} \mathbb{S H}\right)+\mathbf{H} \times \nabla \times\left(\mathbf{F}+\frac{4}{5} \mathbb{S H}\right)\right]$,

where $\mathbf{F}=(\nabla \times \mathbf{u} / 2) \times \mathbf{H}$ and $s_{N}$ is the Niklas parameter [Eq. (11)]. $p_{M}$ is the dynamic pressure incorporating all magnetic terms that can be expressed as gradients including the corresponding part of the Kelvin force $(M \cdot \nabla) H$ resulting from the equilibrium magnetization; $p_{M}=p-\tilde{p}$, with $2\left(\mathbf{M}_{e q} \cdot \nabla\right) \mathbf{H}=\chi\left(H^{2}\right) \nabla H^{2}=: \frac{\partial \widetilde{p}}{\partial H^{2}} \nabla H^{2}=\nabla \widetilde{p}$. To the leading order, the internal magnetic field in the ferrofluid can be approximated by the externally imposed field [28], which is reasonable for obtaining the dynamical solutions of the magnetically driven fluid motion. Eq. (8) can then be simplified as

$$
\begin{aligned}
\left(\partial_{t}+\mathbf{u} \cdot \nabla\right) \mathbf{u}-\nabla^{2} \mathbf{u}+\nabla p_{M} & \\
=s_{N}^{2}\left\{\nabla^{2} \mathbf{u}-\frac{4}{5}[\nabla \cdot(\mathbb{S H})]-\mathbf{H}\right. & \\
\times\left[\frac{1}{2} \nabla \times(\nabla \times \mathbf{u} \times \mathbf{H})-\mathbf{H} \times\left(\nabla^{2} \mathbf{u}\right)+\right. & \frac{4}{5} \nabla \\
& \times(\mathbb{S H})]\} .
\end{aligned}
$$

This way, the effect of the magnetic field and the magnetic properties of the ferrofluid on the velocity field can be characterized by a single 
parameter, the magnetic field or the Niklas parameter [8]:

$s_{N}^{2}=s_{x}^{2}+s_{z}^{2}$,

with

$s_{x}^{2}=\frac{2(2+\chi) H_{x} c_{N}}{(2+\chi)^{2}-\chi^{2} \eta^{2}}, s_{z}^{2}=H_{z} c_{N}$.

Note that in this study we are only investigating pure axial or pure transverse magnetic field, which means that either $s_{x}=0$ or $s_{z}=0$, respectively.

Worth to emphasize the limitation of the Niklas approximation, which results from the stationary case and for small deviations of the magnetization near equilibrium, from one relaxation equation with one relaxation time. Thus the relaxation into equilibrium is determined by a relaxation constant that can depend on the magnetic field.

\section{A.2. Numerical methods}

The ferrohydrodynamical equations of motion Eq. (8) can be solved $[13,17,28]$ by combining a standard, second-order finite-difference scheme in $(r, z)$ with a Fourier spectral decomposition in $\theta$ and (explicit) time splitting. The variables can be expressed as

$f(r, \theta, z, t)=\sum_{m=-m_{\max }}^{m_{\max }} f_{m}(r, z, t) e^{i m \theta}$,

where $f$ denotes one of the variables $\{u, v, w, p\}$. For the parameter regimes considered, the choice $m_{\max }=16$ provides adequate accuracy. We use a uniform grid with spacing $\delta r=\delta z=0.02$ and time steps $\delta t<1 / 3800$. For diagnostic purposes, we also evaluate the complex mode amplitudes $f_{m, n}(r, t)$ obtained from a Fourier decomposition in the axial direction:

$f_{m}(r, z, t)=\sum_{n} f_{m, n}(r, t) e^{i n k z}$,

where $k=2 \pi d / \lambda$ is the axial wavenumber.

For code validation we also compared SPI solutions with experiments $[15,43]$ and previous numerical simulations. In addition the non-linear primary bifurcating solutions (TVF and SPI) were compared with the respective stability boundaries of the linearized NSE obtained by a shooting method [12]. The bifurcation thresholds for both primary vortex structures in TCS were found to lie about $0.5 \%$ below the respective linear stability thresholds, whereby the mesh size has been continuously reduced towards finer discretizations until no further variation in the results and thus deviation from the linear thresholds could be detected. In addition further investigation of the nonlinear solutions change when varying $m_{\max }$ and/or the grid spacing revealed that typical SPI frequencies have an error of less than about $0.2 \%$. Time steps were always well below the von Neumann stability criterion and by more than a factor of 3 below the Courant-Friederichs-Lewy criterion.

Note that for a ferrofluids in presence of a transverse magnetic field $\left(s_{x} \neq 0\right)$, the symmetries present in classical TCS (arbitrary rotations about the axis and the reflections about axial mid-height) are broken and the flow is inherently three-dimensional for any combination of non-zero values of the parameters $R e_{i}, R e_{o}$ and $s_{x}[13,16,28,17]$. A study of pVs under oblique magnetic fields $\left(s_{x} \neq 0 \neq s_{z}\right)$ results in even complexer scenarios due to further non-linear mode-interactions $[13,34]$.

\section{Appendix B. Supplementary data}

Supplementary data associated with this article can be found, in the online version, athttps://doi.org/10.1016/j.jmmm.2021.167769.

\section{References}

[1] G.I. Taylor, Stability of a viscous liquid contained between two rotating cylinders, Philos. Trans. R. Soc. London A 223 (1923) 289.

[2] P. Chossat, G. Iooss, The Couette-Taylor Problem, Springer, Berlin, 1994.

[3] R.C. DiPrima, H.L. Swinney, Instabilities and transition in flow between concentric rotating cylinders, in: H.L. Swinney, J.G. Gollub (Eds.), Hydrodynamic Instabilities and the Transition to Turbulence, number 45 in Topics in Applied Physics, Springer, Berlin, 1985.

[4] C.D. Andereck, S.S. Liu, H.L. Swinney, Flow regimes in a circular Couette system with independently rotating cylinders, J. Fluid Mech. 164 (1986) 155.

[5] M. Golubitsky, W.F. Langford, Pattern formation and bistability in flow between counterrotating cylinders, Physica D 32 (1988) 362.

[6] M.I. Shliomis, Effective Viscosity of Magnetic Suspensions, Sov. Phys. JETP 34 (1972) 1291-1294.

[7] R.E. Rosensweig, Ferrohydrodynamics, Cambridge University Press, Cambridge, 1985.

[8] M. Niklas, Influence of magnetic fields on Taylor vortex formation in magnetic fluids, Z. Phys. B 68 (1987) 493

[9] O. Ambacher, S. Odenbach, K. Stierstadt, Rotational viscosity in ferrofluids, Z. Phys. B 86 (1992) 29.

[10] S. Odenbach, Magnetoviscous Effects in Ferrofluids, volume m71 of Lecture Notes in Physics. Springer, Berlin (2002).

[11] S. Odenbach (Ed.), Ferrofluids - Magnetically controllable Fluids and their Applications, Lecture Notes in Physics, volume 594, Springer, Berlin, 2002.

[12] A. Leschhorn, M. Lücke, C. Hoffmann, S. Altmeyer, Stability of circular Couette flow of a ferrofluid in an axial magnetic field: Influence of polydispersity, Phys. Rev. E 79 (2009) 036308.

[13] S. Altmeyer, C. Hoffmann, A. Leschhorn, M. Lücke, Influence of homogeneous magnetic fields on the flow of a ferrofluid in the Taylor-Couette system, Phys. Rev. E 82 (2010) 016321.

[14] S. Altmeyer, Pattern Formation and Stability in Magnetohydrodynamics 'Interaction of Magnetic Fields on Ferrofluidic Taylor-Couette Flow, IntechOpen 10 (2018) 5772.

[15] M. Reindl, S. Odenbach, Influence of a homogeneous axial magnetic field on Taylor-Couette flow of ferrofluids with low particle-particle interaction, Expts. Fluids 50 (2011) 375.

[16] M. Reindl, S. Odenbach, Effect of axial and transverse magnetic fields on the flow behavior of ferrofluids featuring different levels of interparticle interaction, Phys. Fluids 23 (2011) 093102.

[17] S. Altmeyer, J. Lopez, Y. Do, Effect of elongational flow on ferrofuids under a magnetic field, Phys. Rev. E 88 (2013) 013003.

[18] S. Altmeyer, Y.-H. Do, Y.-C. Lai, Transition to turbulence in Taylor-Couette ferrofluidic flow, Sci. Rep. 5 (2015) 10781.

[19] S. Altmeyer, Y.-H. Do, Y.-C. Lai, Magnetic field induced flow reversal in a ferrofluidic Taylor-Couette system, Sci. Rep. 5 (2015) 18589.

[20] S. Altmeyer, Non-linear dynamics and alternating 'flip' solutions in ferrofluidic Taylor-Couette flow, J. Magn. Magn. Mater. 452 (2018) 427.

[21] R. Tagg, The Couette-Taylor Problem, Nonlinear Sci. Today 4 (1994) 1.

[22] T. Benjamin, Bifurcation phenomena in steady flows of a viscous fluid. I. Theory. Philo. Trans. Roy. Soc. A p. 1 (1978).

[23] W. Edwards, S. Beane, S. Varma, Onset of wavy vortices in the finite-length Couette-Taylor problem, Phys. Fluids 3 (1991) 1510.

[24] O. Czarny, E. Serre, P. Bontoux, R. Lueptow, Interaction between Ekman pumping and the centrifugal instability in Taylor-Couette flow, Phys. Fluids 15 (2003) 467.

[25] S. Altmeyer, C. Hoffmann, M. Heise, J. Abshagen, A. Pinter, M. Lücke, G. Pfister, End wall effects on the transitions between Taylor vortices and spiral vortices, Phys. Rev. E 81 (2010) 066313.

[26] J. Linke, S. Odenbach, Anisotropy of the magnetoviscous effect in a cobalt ferrofluid with strong interparticle interaction, J. Magn. Magn. Mater. 396 (2015) 85.

[27] M. Niklas, H. Müller-Krumbhaar, M. Lücke, Taylor-vortex flow of ferrofluids in the presence of general magnetic fields, J. Magn. Magn. Mater. 81 (1989) 29.

[28] S. Altmeyer, J. Lopez, Y. Do, Influence of an inhomogeneous internal magnetic field on the flow dynamics of ferrofluid between differentially rotating cylinders, Phys. Rev. E 85 (2012) 066314.

[29] C. Hoffmann, S. Altmeyer, M. Heise, J. Abshagen, G. Pfister, Axisymmetric propagating vortices in centrifugally stable Taylor-Couette flow, J. Fluid Mech. 728 (2013) 458.

[30] T. Ilzig, K. Stöckel, S. Odenbach, Experimental Investigations on the Effect of Axial Homogenous Magnetic Fields on Propagating Vortex Flow in the Taylor-Couette System, Materials 12 (2019) 4027.

[31] M. Nagata, On wavy instabilities of the Taylor-vortex flow between corotating cylinders, J. Fluid Mech. 88 (1988) 585.

[32] S.T. Wereley, R.M. Lueptow, Spatio-temporal character of non-wavy and wavy Taylor-Couette flow, J. Fluid Mech. 364 (1998) 59.

[33] C.A. Jones, The transition to wavy Taylor vortices, J. Fluid Mech. 175 (1985) 135

[34] S. Altmeyer, Propagating vortices in ferrofluidic Couette flow under magnetic fields - part II: oblique orientated fields. To be submitted to J. Magn. Magn. Mater. (2020).

[35] H.W. Müller, M. Liu, Structure of ferrofluid dynamics, Phys. Rev. E 64 (2001) 061405. 
[36] P. Langevin, Magnétisme et théorie des électrons, Annales de Chemie et de Physique 5 (1905) 70.

[37] S. Altmeyer, Y.-H. Do, Y.-C. Lai, Dynamics of ferrofluidic flow in the Taylor-Couette system with a small aspect ratio, Sci. Rep. 7 (2017) 40012.

[38] F. Marques, J.M. Lopez, Onset of three-dimensional unsteady states in small-aspect ratio Taylor-Couette flow, J. Fluid Mech. 561 (2006) 255

[39] J. Abshagen, M. Heise, G. Pfister, T. Mullin, Multiple localized states in centrifugally stable rotating flow, Phys. Fluids 22 (2010) 021702.
[40] S. Altmeyer, Y. Do, F. Marquez, J.M. Lopez, Symmetry-breaking Hopf bifurcations to 1-, 2-, and 3-tori in small-aspect-ratio counterrotating Taylor-Couette flow, Phys. Rev. E 86 (2012).

[41] J. Embs, H.W. Müller, C. Wagner, K. Knorr, M. Lücke, Measuring the rotational viscosity of ferrofluids without shear flow, Phys. Rev. E 61 (2000) R2196.

[42] S. Odenbach, H.W. Müller, Stationary off-equilibrium magnetization in ferrofluids under rotational and elongational flow, Phys. Rev. Lett. 89 (2002) 037202.

[43] A. Schulz and G. Pfister, Physics of Rotating Fluids Lecture Notes in Physics, Ed. C. Eghers and G. Pfister, Springer, Berlin, p. 37 (2000). 